\title{
Effect of variable nutrient supply on fatty acid composition of phytoplankton grown in an enclosed experimental ecosystem
}

\author{
P. Mayzaud ${ }^{1}$, H. Claustre ${ }^{2}$, P. Augier ${ }^{2}$ \\ ${ }^{1}$ INRS-Océanologie, 310 Allée des Ursulines, Rimouski, Québec, Canada G5L 3A1 \\ ${ }^{2}$ C.E.R.O.V., Station Zoologique, F-06230 Villefranche-sur-Mer, France
}

\begin{abstract}
Outdoor experimental tanks were used to simulate phytoplankton blooms in natural seawater from the bay of Villefranche-sur-Mer (France), during March-April 1984. External temperature and light intensity were respectively monitored every $4 \mathrm{~h}$ and daily. Nutrient conditions were followed daily and maintained by a cycle of dilutions with fresh enriched seawater. Six different cycles of phytoplankton production were followed. They differed by the dilution rates used. Nutrient uptake, phytoplankton composition, biochemical composition and detailed lipid composition of the phytoplankton were followed throughout each growth cycle. Results showed a clear difference between the first cycle, which lasted $7 \mathrm{~d}$, and subsequent ones which lasted only from 27 to $51 \mathrm{~h}$. In terms of species composition, the initial body of water comprised a complex assemblage of species (flagellates, dinoflagellates, diatoms, microzooplankton) which were gradually replaced by a community of diatoms dominated by Skeletonema costatum, Nitzschia sp. and Chaetoceros sp. Total fatty acid changes reflected the progressive changes in species composition (inverse variation of C16 and C18 polyunsaturated fatty acids) and to a minor extent the nutrient regime. In terms of fatty acid composition of the different lipid classes the transition from a flagellate to a diatom population appears to be the key factor influencing all categories in a similar way. Nutrient regime affected essentially the neutral lipid composition while changes in physiological state were reflected in the polar lipid composition (phospholipids, monogalactosyl diglycerides [MGDG], digalactosyl diglycerides). Probable light limitation during the fourth growth cycle may explain some of the changes in fatty acid composition at the MGDG level. In all cases, transient saturation-desaturation processes of the membrane lipids seems at the heart of the acclimation dynamics.
\end{abstract}

\section{INTRODUCTION}

The complexity of the natural environment makes it difficult to clarify the processes involved in regulation of phytoplankton fatty acid composition. The few studies on temporal changes of natural particulate assemblages have shown that both fatty acid and sterol components are related in various degrees to physical, chemical and physiological parameters (Jeffries 1970, 1972, Schultz 1974, Goutx \& Saliot 1980, Kattner et al. 1983, Mayzaud et al. 1989) whose influences are often compounded. The use of laboratory cultures of phytoplankton has been the alternate choice to ascertain patterns of fatty acid biosynthesis and the importance of individual environmental variables (see reviews by Wood 1974, Pöhl \& Zurheide 1979), but cannot be directly extrapolated to natural conditions. Recent studies by Morris (1984) and Morris et al. (1985) of phytoplankton blooms produced in large-volume experimental ecosystem have introduced an interesting alternative for investigating the nature of biochemical changes which occur at various stages of phytoplankton growth.

Most studies using experimental ecosystems or mesocosms (Brockmann et al. 1977, Grice \& Reeve 1982) have focused on the time course of an undisturbed, well-characterized isolated water body. Though the progressive nutrient depletion observed under such conditions is representative of wellstratified surface water layers, it does not take into account the frequent mixing of the water column. In the present study, we report the fatty acid composition of the component lipid classes of phytoplankton populations subjected to pulses of nutrient supply. Such data 
should yield important information on the acclimation processes of phytoplankton cells in a periodically changing environment.

\section{MATERIAL AND METHODS}

Experimental ecosystem. The experimental system consisted of a $2.2 \mathrm{~m}^{3}$ plastic glassfiber-polyester tank fitted with a transparent $3 \mathrm{~mm}$ thick plastic cover to exclude atmospheric contaminants. This cover reduced the quantity of light by $23 \%$ but did not affect the spectral composition. An airlift system was used to ensure the homogeneity of the water column and dissipate the slight increase in temperature produced by the cover. The system was placed outside and exposed to natural changes of light and temperature.

Seawater for the experiment was pumped from a depth of $3 \mathrm{~m}$ in the bay of Viliefranche-sur-Mer, France, filtered through a $150 \mu \mathrm{m}$ bolting cloth to remove large zooplankton and debris and enriched with nutrients (nitrate, phosphate, silicate) in the proportions suggested by Redfield (1934) and Harris \& Riley (1956), i.e. 12. 1:8

Throughout the experiment, light, temperature and nutrient levels were monitored continuously using probes and a 3-way Technicon Autoanalyser II. Nitrate, nitrite, phosphate and silicate were measured every 30 min following Tréguer \& Le Corre (1974).

Biomass control and sampling. Particulate volume chlorophyll, total proteins and lipids were monitored daily. Known volumes were filtered on prebaked GF/C filters and frozen $\left(-70^{\circ} \mathrm{C}\right)$ until analysis. Particle concentrations and volume at sizes ranging from 1 to $60 \mu \mathrm{m}$ equivalent diameter were estimated using a PC 230 Hiac counter. Particulate chlorophyll and phaeopigment were measured by fluorometry according to the method of Yentsch \& Menzel (1963) as modified by Holm-Hansen et al. (1965). Total proteins were estimated according to Lowry et al. (1951) while

Table 1 Solvent system for the separation of the mann lipid classes. MGDG: monogalactosyl diglycerıdes; DGDG: digalactosyl diglycerides; SQDG: sulfoquinovosyl diglycerides: $\mathrm{MeOH}$ : methanol

\begin{tabular}{|llc|}
\hline Lipid classes & Solvent system & $\begin{array}{c}\text { Column } \\
\text { volume }\end{array}$ \\
\hline Hydrocarbons & Hexane & 1 \\
Neutral lipids & Chioroform & 10 \\
MGDG & Chloroform:acetone & 8 \\
& $60: 40$ & 25 \\
DGDG + SQDG & Acetone & 10 \\
Phospholipids & MeOH:ammona & \\
& $90: 10$ & \\
& & \\
\hline
\end{tabular}

total lipids were measured gravimetrically after extraction according to Bligh \& Dyer (1959). Nutrients were analyzed in $200 \mathrm{ml}$ fractions of filtered water. Analyses were done in duplicate.

Lipid analysis. Aliquots of 100 to 200 1, depending on the growth phase of the phytoplankton population, were filtered on $12.5 \mathrm{~cm}$ GF/C filters using a stainlesssteel holder, a peristaltic pump equipped with silicone tubing and a volume-meter. All samples were extracted within the same day and the lipid stored in chloroform at $-70^{\circ} \mathrm{C}$ under nitrogen.

Lipid classes were separated by column chromatography on silica gel (100 to $200 \mu \mathrm{m}$ mesh) using a system of solvents of increasing polarity (Table 1) adapted from Vorbeck \& Marinetti (1965). A $30 \mathrm{ml}$ glass column $(26.5 \mathrm{~cm}$ height, $1.2 \mathrm{~cm}$ diameter) equipped with a fritted disc was used with a mean flow of

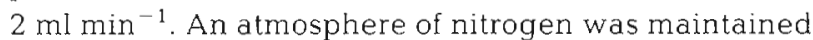
at all time. Possible cross contaminations between classes was checked by thin layer chromatography (TLC) eluted by hexane:diethyl ether:acetic acid $(80: 20: 1)$ for neutral lipids, chloroform:methanol-water (85:30:1) for glycolipids and chloroform:methanol: ammonia $(65: 35: 5)$ for phospholipids. The phospholipids, glycolipids and neutral lipids were methylated using $7 \% \mathrm{BF}_{3}$ in methanol (Morrison \& Smith $1964\}$.

Gas liquid chromatography (GLC) of methyl esters was carried out with a Perkin-Elmer Sigma 2 chromatograph equipped with a flame ionization detector and a Spectra-Physics SP4000 integrator. The wall-coated open tubular (capillary) columns used were of stainless steel, $48 \mathrm{~m}$ in length $\times 0.25 \mathrm{~mm}$ internal diameter, coated with SILAR 5CP or Apiezon L. The columns were operated isothermally at $165^{\circ} \mathrm{C}$ (SILAR 5CP) and $190^{\circ} \mathrm{C}$ (Apiezon L). Helium was used as carrier gas (Mayzaud \& Ackman 1976) at 60 psig (SILAR 5CP) and 80 psig (Apiezon L). Injector and detector were maintained at $250^{\circ} \mathrm{C}$. In addition to the examination of esters as recovered, part of all ester samples were completely hydrogenated and the products examined quantitatively and qualitatively by GLC. Results are given to 2 decimal places to permit the inclusion of minor components, but this does not imply this order of accuracy. Major components ( $>10 \%$ ) should be accurate to $\pm 5 \%$, moderate size components ( 1 to $9 \%$ ) to $\pm 10 \%$ and minor components $(<1 \%)$ to up to $\pm 50 \%$

Nomenclature. A shorthand notation for unsaturated acids is employed in this paper as follows: chain length : number of double bonds and number of carbon atoms from the centre of the ultimate double bond to the terminal methyl group (e.g. $20: 5(1) 3$ or $20: 5 n-3$ indicate 20 carbons, 5 double bonds, ultimate double bond 3 carbons from the terminal methyl group). The assumption is that all bonds are cis and are methylene- 
interrupted. The conventional system of numbering from the carboxyl group uses a $\Delta$ notation and makes the same assumption unless otherwise indicated. PUFA (polyunsaturated fatty acids) describes all acids with more than 2 double bonds and HUFA (highly unsaturated fatty acids) those acids wiht more than 5 double bonds.

Statistical treatment. Because of the overwhelming amount of data generated by GLC analyses, a comparison of their covariation is not possible by classical bivariate approach. In the present study, correlation analysis would describe the dominant temporal association rather than the day-to-day variability and would not take into account all the interrelations of the variables. A multivariate approach is more satisfactory to compare all data sets in a 3-dimensional space as it allows extraction of the major trends of variability of a set of samples for all the descriptors (fatty acids). A principal component analysis (PCA) was performed on the correlation matrix of fatty acid data according to the approach described by Legendre \& Legendre (1984) and the application to fatty acid data of Mayzaud et al. (1989). Briefly, a correlation matrix computed from the original data set is used to obtain a set of eigenvalues associated with a similar number of eigenvectors which define a new orthogonal space. The new axes are linear combinations of the initial variables and are called principal components. The eigenvalues represent the amount of variance encountered by the corresponding principal components. The elements of the resulting factor loading matrix are correlations between the original variables (fatty acids) and the principal components. Factor scores which represent the contribution of the observations to the axes are calculated to have a zero mean and unit variance. The factorial axes may be interpreted using the elements of the factor loading matrix and the factor scores of the observations

Once the factorial axes based on the active variables' have been extracted, 'supplementary variables' not used in the determination of the axes may be added to the analysis. These may be located by projecting them on to the factorial planes of the first principal components derived from the active variables only. Formulations for computing supplementary variable projections may be found in Lebart et al. (1977).

In the present study, the aim of the analysis is to understand the sequence of observations based on the similitude of fatty acid spectra. In the projections, fatty acids with similar temporal variation will show similar levels of correlations with the principal components or axes while observations with similar fatty acid profiles will display similar scores. Because the principal components correspond to decreasing amounts of the total variance, interpretation is usually based on a limited number (usually 3) which account for most of the variance recorded. Based on the significance of the groups of descriptors which define the axes, inferences on the ecological or physiological meaning of the axes can be drawn. Each level of interpretation can then be associated to the degree of variance of the corresponding principal component (or axis) and/or projection plane. Once the variance of the first axis has been interpreted, the remainder can be explained independently from the associations along the second and subsequent axes.

\section{RESULTS}

\section{Nutrient regime and environmental parameters}

The experiment was initiated on 30 March 1983 and lasted for $16 \mathrm{~d}$. During this period, ambient light was monitored daily and the water temperature every $4 \mathrm{~h}$. Average daily water temperature varied over a range of 3 to $5^{\circ} \mathrm{C}$ (Fig. 1 top diurnally between 13 and $19^{\circ} \mathrm{C}$
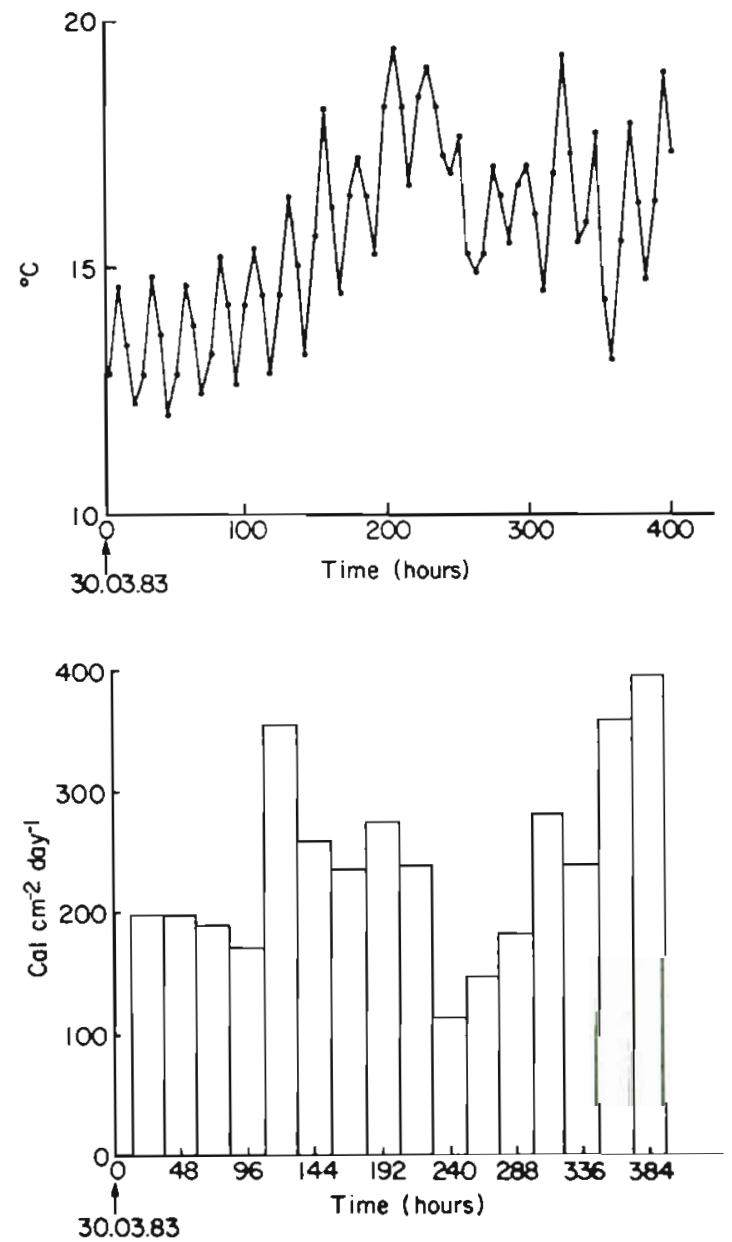

Fig. 1. Changes in temperature (top) and light intensity (bottom) in the experimental tank. Temperature was measured at mid-depth in the water column and light at the surface 
with a maximum around Day 9. Light showed 2 periods of high intensity around Days 5 and 16 and one period of low intensity on Day 10 (Fig. 1 bottom). In such a case, the growth of phytoplankton could have been partially limited during the period of minimum values but not totally inhibited

Changes in nutrient concentrations were controlled, over time, both by the cellular uptake and by the cycles of dilution with fresh enriched seawater. Six production cycles were considered and the time of dilution was set to prevent severe limitations. Different dilution rates were used (Table 2) and the concentrations of each nutrient adjusted as a function of the specific uptakes

Table 2. Rate of dilution at the end of each growth cycle. Dilution rate $=$ volume of water added/total volume

\begin{tabular}{|cc|}
\hline Cycle no. & $\begin{array}{c}\text { Dilution rate } \\
(\%)\end{array}$ \\
\hline 1 to 2 & 50 \\
2 to 3 & 50 \\
3 to 4 & 66 \\
4 to 5 & 66 \\
5 to 6 & 66 \\
\hline
\end{tabular}

recorded during the previous cycle. Thus, the decision to end a growth cycle was based on nitrate exhaustion rather than on biomass concentration. The results presented in Fig. 2 suggest a clear difference between the first cycle and the subsequent ones. The first one lasted for $174 \mathrm{~h}(7.3 \mathrm{~d})$ and displayed relatively slow uptake of nitrates, phosphates and silicates. The following cycles lasted for 27 to $51 \mathrm{~h}$ and revealed a high consumption level of all 3 nutrients. Nitrites showed an increasing trend through the $16 \mathrm{~d}$ experiment, but remained at very low levels $\left(<0.6 \mu \mathrm{g}\right.$-at $\left.\mathrm{l}^{-1}\right)$.

\section{Changes in particulate biomass}

Biomass changes were followed in terms of chlorophyll, total proteins and total volumes. As shown in Fig. 3, all 3 parameters displayed a similar pattern of variations throughout the 6 growth cycles. Except during the first cycle, chlorophyll a appeared to dominate over phaeopigments suggesting a dominance of phytoplankton cells with little detritus fraction. Microscopic observations confirmed the dominance of a small number of diatom species including Nitzschia sp., Chaetoceros sp. and Skeletonema costatum. During the first cycle, the composition of the experimental assemblage of particules was more complicated as it reflected the natural diversity of the bay water used and comprised. flagellates, dinoflagellates, diatoms, microzooplankton and detritus. As expected each exponential growth phase was characterized by an initial dominance of protein synthesis followed by an increase of particulate volume.

In terms of biomass, each cycle displayed a different production level. As indicated in Table 3 , the proteinbased growth rates showed a general increase throughout the experiment. The differences are related to the level of light intensity and to the initial biomass left after each dilution. The low light explains the reduced growth rate during Cycle 4 while the larger dilution $(66 \%)$ seemed to promote higher growth rates.
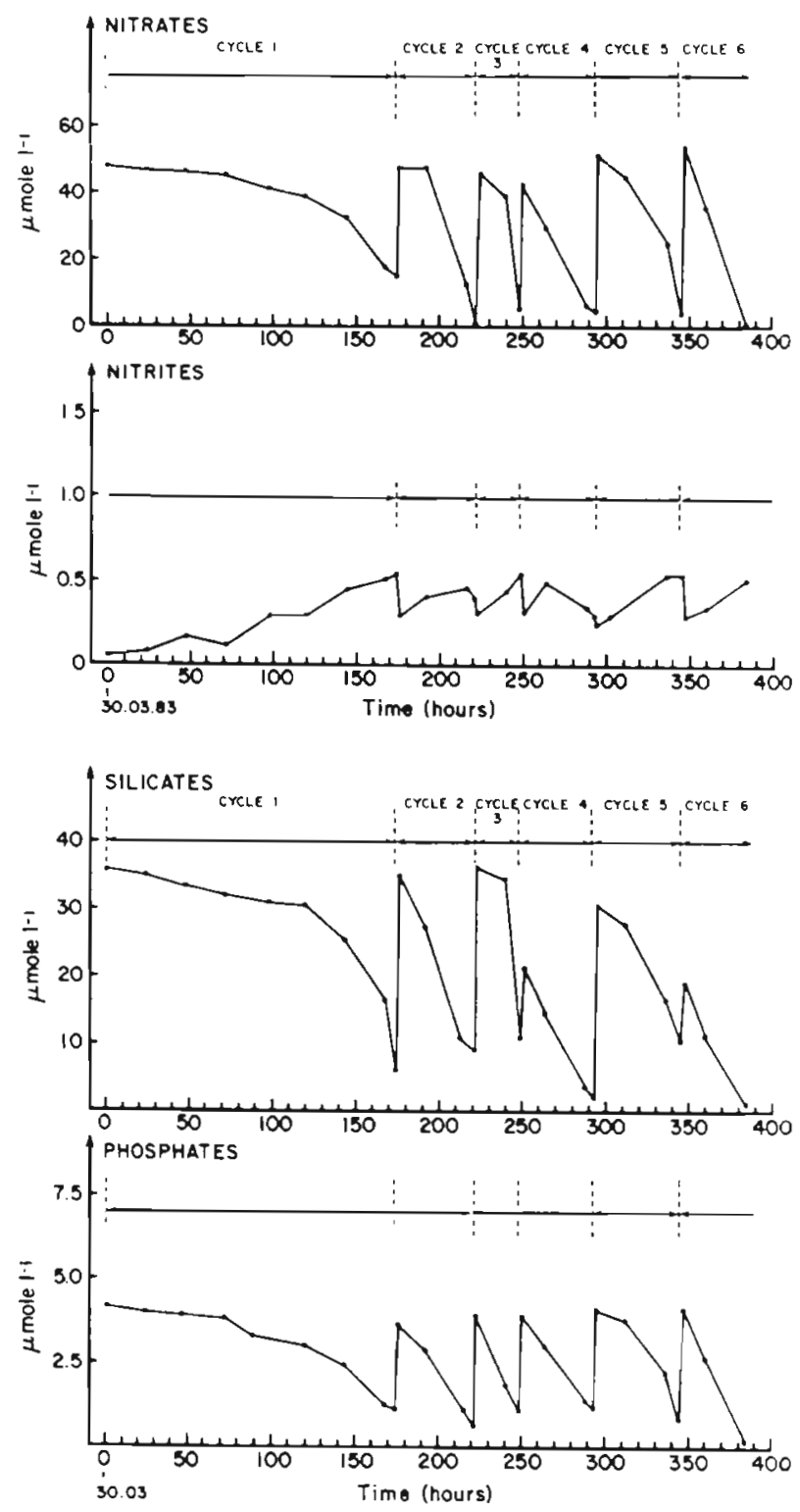

Fig. 2. Temporal changes in concentration of inorganic nutrient throughout the different dilution cycles 
Fig. 3. Time course changes of particulate volume, chlorophyll, phaeopigment and protein concentration during the successive growth cycles. Arrows indicate those samples when lipid analyses were performed
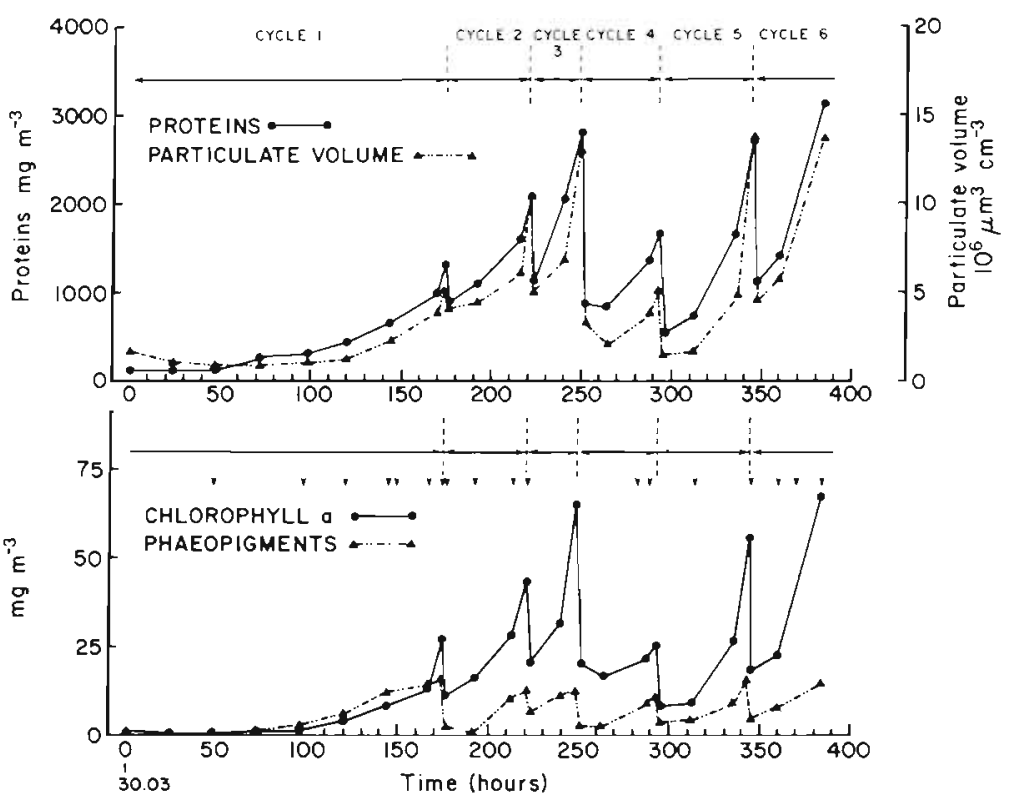

\section{Changes in total lipid and fatty acids}

The lipid level of the total phytoplankton populations (Fig. 4) rose slowly during the first 4 growth cycles. It increased sharply at the end of the fifth cycle and dropped after dilution to reach a maximum value by the end of Cycle 6 . The lipid: total volume ratio displayed an expected rhythmicity with maximum values during the early exponential growth phase (Fig. 4) suggesting that lipids accumulated before the biomass maximum expressed either in terms of volume or chlorophyll.

The fatty acid composition of the total lipids throughout the experiment is given in Table 4. Four days after the beginning of the experiment, the total lipids were dominated by a group of 6 fatty acids $(22: 6 \omega 3,18: 4 \omega 3$, $18: 1 \omega 7,16: 1 \omega 7,16: 0,14: 0)$, occurring in similar proportions and characteristic of the natural population trapped before the addition of nutrients. At the end of the sixth growth cycle, both palmitoleic acid $(16: 107)$ and eicosapentaenoic acid (20:5w3) had increased considerably and made up close to $40 \%$ of the total

Table 3. Phytoplankton growth rate computed in terms of proteins for each cycle

\begin{tabular}{|cc|}
\hline $\begin{array}{c}\text { Growth } \\
\text { cycle no. }\end{array}$ & $\begin{array}{c}\text { Exponential growth } \\
\text { rate }\left(\mathrm{d}^{-1}\right)\end{array}$ \\
\hline $1(0-174 \mathrm{~h})$ & 0.37 \\
$2(175-221 \mathrm{~h})$ & 0.46 \\
$3(222-249 \mathrm{~h})$ & 0.77 \\
$4(250-293 \mathrm{~h})$ & 0.56 \\
$5(294-345 \mathrm{~h})$ & 0.93 \\
$6(346-384 \mathrm{~h})$ & 0.79 \\
\hline
\end{tabular}

fatty acids. Saturated acids displayed no clear trend of variation throughout the experiment while opposing trends characterized the relative concentrations of $\mathrm{C} 16$ and C18 polyunsaturates (Fig. 5). Increasing levels of $16: 2 \omega 4,16: 3 \omega 4,16: 4 \omega 1$ and decreasing levels of $18: 2 \omega 6,18: 3 \omega 3,18: 4 \omega 3$ and $18: 5 \omega 3$ explained this pattern of variation.

To understand the influence of successive nutrient impulses on the fatty acid constituents, a correlation matrix was subjected to PCA. Three axes were needed to explain $83 \%$ of the total variance (Fig. 6). The first 2 axes account respectively for $56.3 \%$ and $16.1 \%$ of the

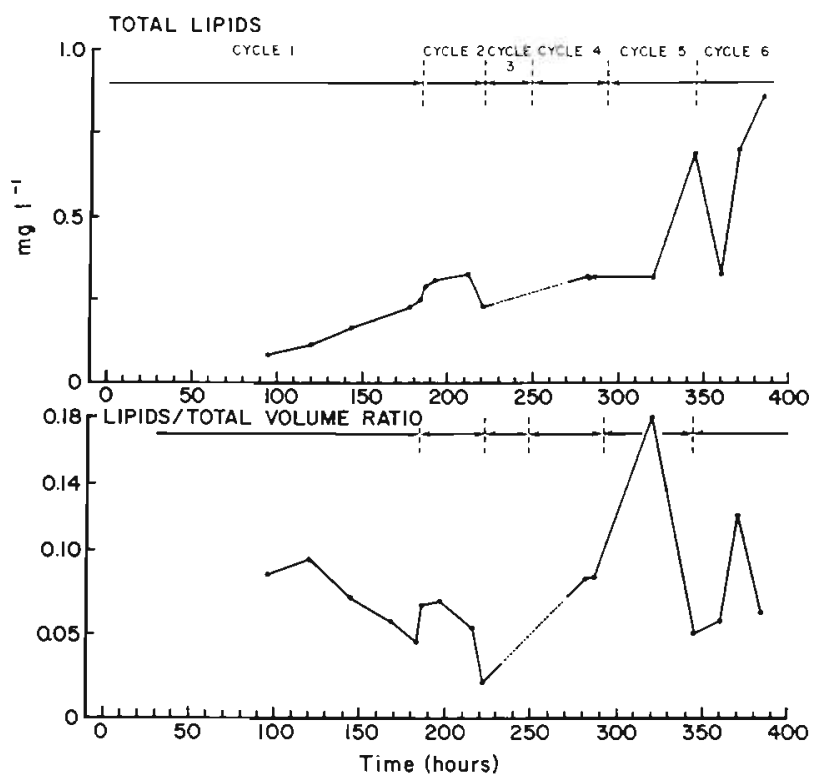

Fig. 4. Changes in particulate lipid concentration (top) and the lipid: particulate volume ratio (bottom). Dotted lines indicate a lack of data for that growth cycle 


\begin{tabular}{|c|c|c|c|c|c|c|c|}
\hline 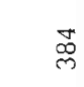 & 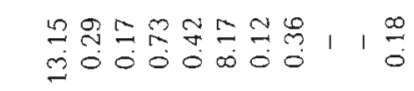 & 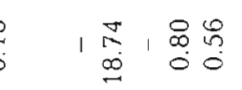 & 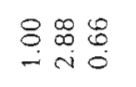 & 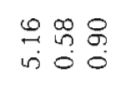 & 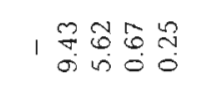 & 色吕 & $\stackrel{0}{0}$ \\
\hline 点 & 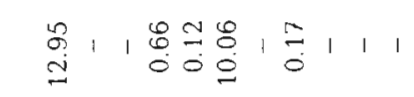 & 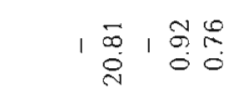 & 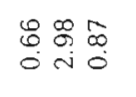 & 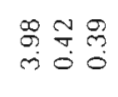 & 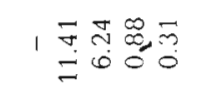 & $\begin{array}{l}\bar{N} \tilde{O} \\
0 \\
0\end{array}$ & $\stackrel{8}{\leftrightarrow}$ \\
\hline $\begin{array}{l}\infty \\
\stackrel{0}{n}\end{array}$ & 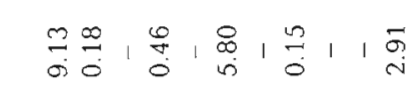 & 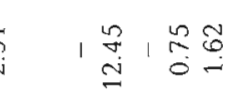 & 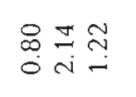 & $\begin{array}{ll}R & m \\
\sim & m \\
0 & m \\
0\end{array}$ & 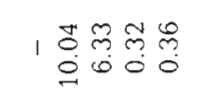 & 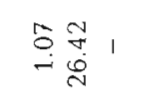 & $\begin{array}{l}\stackrel{0}{\infty} \\
\stackrel{0}{0}\end{array}$ \\
\hline 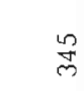 & 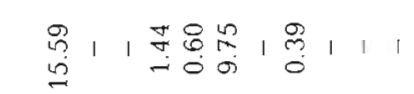 & 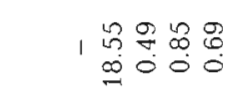 & 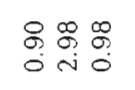 & 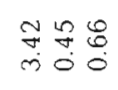 & 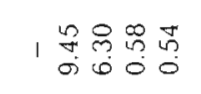 & 焉 & $\stackrel{\llcorner}{\leftrightarrow}$ \\
\hline ్ㅛ & 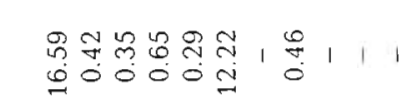 & 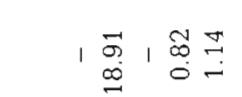 & Wֶ. & 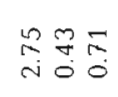 & | & 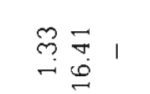 & $\begin{array}{l}\infty \\
i \\
\text { in }\end{array}$ \\
\hline$\stackrel{\infty}{\sim ్ య ~}$ & 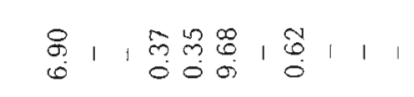 & 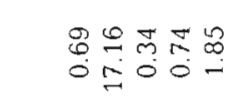 & 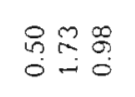 & 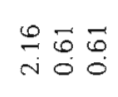 & 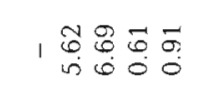 & 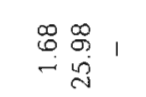 & $\begin{array}{l}\infty \\
\infty \\
\stackrel{\sim}{\ominus}\end{array}$ \\
\hline 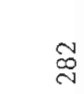 & 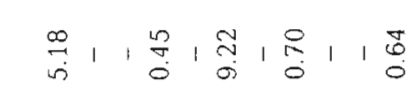 & 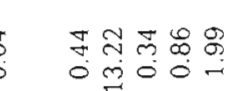 & $\begin{array}{l}\approx \\
\approx \\
0 \\
0\end{array}$ & 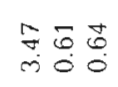 & 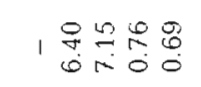 & 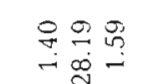 & 药 \\
\hline$\overline{\widetilde{N}}$ & 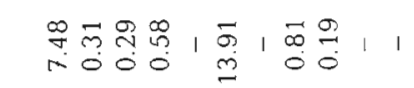 & । & iூ & 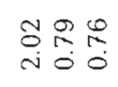 & 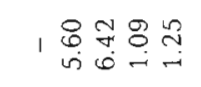 & 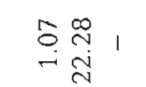 & $\underset{\infty}{\infty}$ \\
\hline$\stackrel{\oplus}{N}$ & 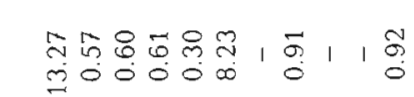 & 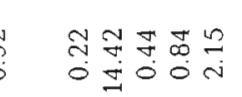 & 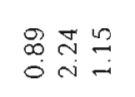 & 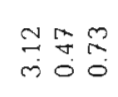 & 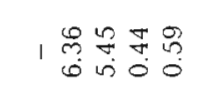 & 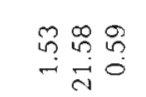 & $\stackrel{\infty}{\circ}$ \\
\hline 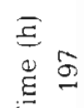 & 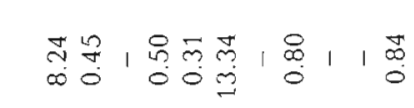 & I & 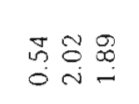 & 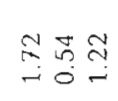 & 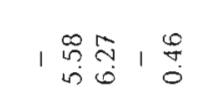 & 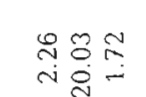 & $\underset{\infty}{\infty}$ \\
\hline 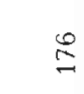 & 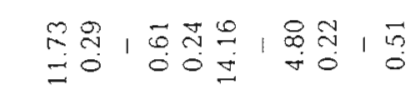 & 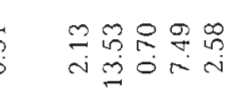 & 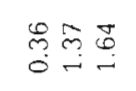 & बृु ले ले & 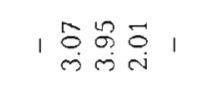 & $\stackrel{\mathscr{N}}{\sim} \stackrel{N}{=} 1$ & $\underset{\infty}{i}$ \\
\hline$\stackrel{2}{=}$ & 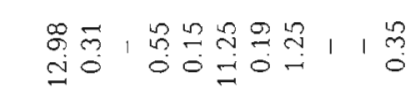 & 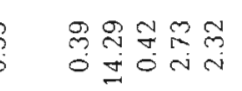 & 용용 & बू. & | & 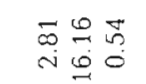 & $\stackrel{g}{\sigma}$ \\
\hline$\stackrel{\mathscr{Q}}{0}$ & 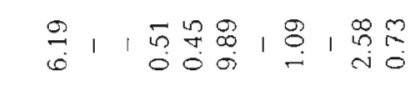 & 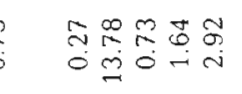 & 䓍尽 & 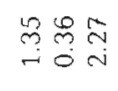 & 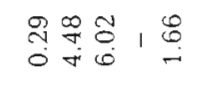 & 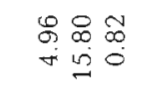 & $\underset{\sim}{\stackrel{\&}{\mathrm{j}}}$ \\
\hline$\underset{g}{g}$ & 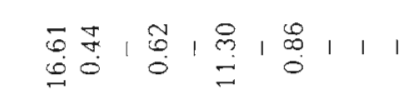 & 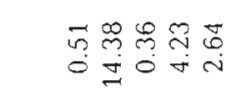 & 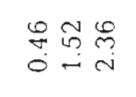 & Oैल & 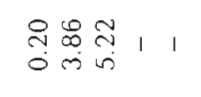 & 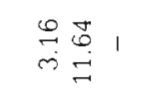 & \& \\
\hline 寻 & 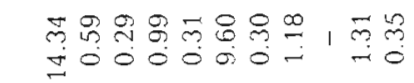 & 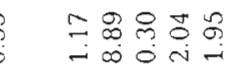 & 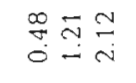 & 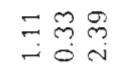 & 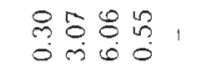 & 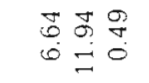 & $\stackrel{\infty}{\stackrel{m}{m}}$ \\
\hline$\stackrel{\Xi}{-}$ & 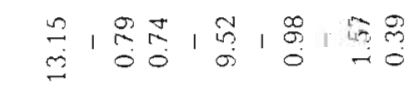 & 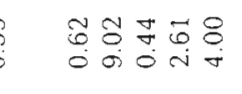 & 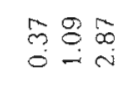 & 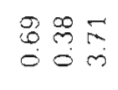 & 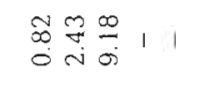 & 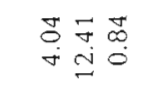 & 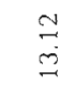 \\
\hline$\tilde{\sigma}$ & 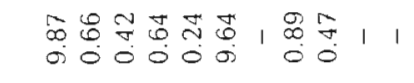 & 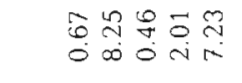 & 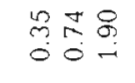 & $\vec{n}$ & 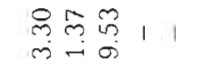 & 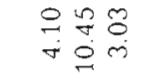 & Nֶ \\
\hline 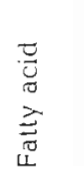 & \multicolumn{7}{|c|}{ 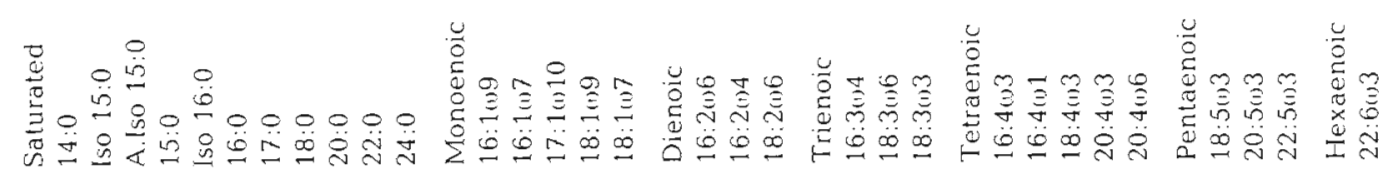 } \\
\hline
\end{tabular}




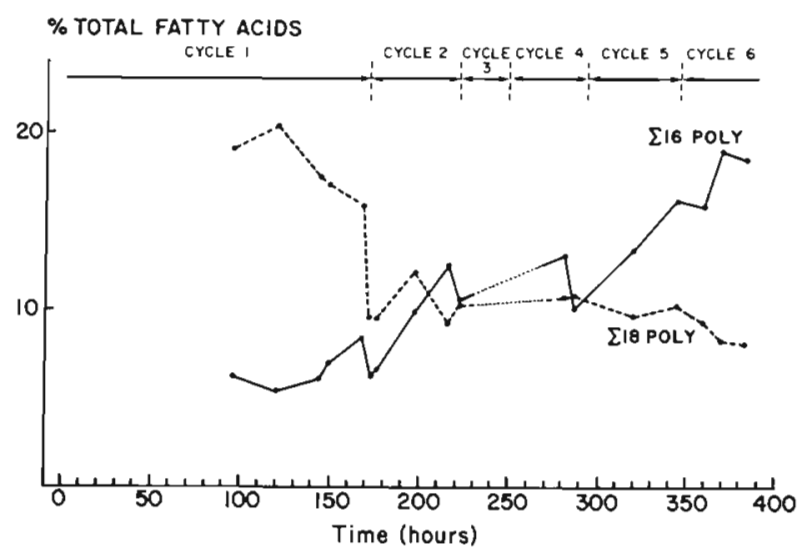

Fig. 5. Temporal changes in percent of total lipid constituted by hexadecanoic and octadecanoic polyunsaturated fatty acids from the different phytoplankton communities throughout the different growth cycles. $\Sigma$ : sum of C16 or C18 polyunsaturated acids

inertia and the third axis for $11 \%$. From the level of correlation between the variables and the principal components, it can be seen that the first axis contrasts the C18 and the C16 polyunsaturated fatty acids (Fig. 6A) which are associated respectively to the first growth cycle and to the remaining five (Fig. 6B). The second axis opposes the polyunsaturated acids to the saturated acids, mainly myristic (14:0) and palmitic (16:0) (Fig. 6A). During the first cycle, this pattern describes the changes occurring during the exponential growth (95 to $176 \mathrm{~h}$ ) while it also illustrates the state of oscillation induced by further dilutions (Fig. 6B). The third axis opposes myristic acid to palmitic acid and 18:3w6 (Fig. 6C) and singles out the second growth period ( 175 to $221 \mathrm{~h}$ ) as the transitional one between the initial population with C18-dominated metabolism and the resulting selected population with C16-dominated metabolism (Fig. 6D). The fatty acids clearly show a time course related mainly to species successions and growth characteristics, and only marginally to the nutrient regime.

\section{Fatty acid composition of major lipid classes}

Because the separation of lipid classes requires large quantities of phytoplankton and is very time-consuming, we selected our samples to be representative of the characteristic features of the first and last growth cycle and of the periods immediately before and after dilution. Depending on the fraction considered 10 to 12 sampling periods were analyzed.

Neutral lipids. The composition is given in Table 5. TLC revealed that triglycerides were the major constituent of this fraction with traces of free fatty acids. At the start of the experiment, the fatty acid composition was dominated by $14: 0,16: 0,16: 107$ and $20: 503$ acids. At the end of the last growth cycle, 20:5 03 and 16:1 $1 \omega^{7}$ showed strong increases at the expense of $16: 0,18: 0,18: 1 \omega 9$ and to minor extent $14: 0,22: 6 \omega 3$ and most $\mathrm{C} 18$ polyunsaturated fatty acids. The increase in $\mathrm{C} 16$ polyunsaturates reflects the change in population despite their lesser importance compared to the total lipid fraction.

A PCA run on a reduced number of major fatty acids showed that the first 3 components can explain $90.5 \%$ of the total variance. The first axis accounts for $55.6 \%$ of the inertia and separates the C18 fatty acids and $22: 6 \omega 3$ from the C16 polyunsaturated acids and 20:5w3 (Fig. 7A). As expected, it corresponds to the occurrence of the initial complex population at the start of the experiment ( 120 to $168 \mathrm{~h}$ ) and its replacement by a diatom dominated population (345 to $384 \mathrm{~h}$ ) (Fig. 7B). The second axis explains $19.4 \%$ of the total variance and opposes $14: 0,15: 0$ and $16: 2 \omega 6$ to $18: 4 \omega 3$ (Fig. $7 \mathrm{~A})$ as well as those sampling times $5 \mathrm{~h}$ before dilution (168, $216 \mathrm{~h}$ ) and $1 \mathrm{~d}$ after (197, $320 \mathrm{~h}$ ) (Fig. 7B). Such statistical discrimination can be better explained by considering the influence of the nutrient depletion which controlled the dilution sequence and the difference in fatty acid between early and late exponential growth phase. The third axis accounts for $15.5 \%$ of the total inertia and opposes $18: 303$ and $14: 0$ to $16: 0$,

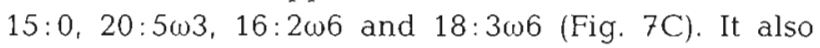
singles out the likely changes in physiological state within the first growth cycle (130 to $168 \mathrm{~h}$ ) and to a minor extent the 2 last cycles (320 to $345 \mathrm{~h}$ and 360 to 384 h) (Fig. 7D).

Monogalactosyl diglycerides (MGDG) displayed a pattern of fatty acid composition quite different from the neutral lipids. Polyunsaturated acids $(16: 3 \omega 4$, $16: 4 \omega 1,18: 4 \omega 3,18: 5 \omega 3$ and $20: 5 \omega 3)$ largely dominated this fraction at the expense of saturated and monoenoic acids (Table 6). Over time the levels of $14: 0,18: 1 \omega 9$, C18 PUFA and 22:6 63 decreased sharply and were replaced by $16: 1 \omega 7$, C16 PUFA and $20: 503$. Hence saturated acids dropped by a factor of 3 while PUFA and monoenoic acids increased by 20 and $50 \%$ respectively (Table 6 ).

Using the same statistical procedure, it can be shown that the first 3 axes derived from a PCA explain $89.7 \%$ of the total variance. The first axis accounts for $67.7 \%$ of the inertia and separates the C16 from the C18 PUFA (Fig. 8A). As noted above, it represents the transition between the initial and the final phytoplankton population (Fig. 8B). The second axis $(14.5 \%$ of the total variance) is mainly defined by 2 saturated acids $(16: 0,18: 0)$, palmitoleid acid $(16: 1 \omega 7)$ and to a minor extent 20:5w3 (Fig. 8A) which reached maximum relative concentrations at $197 \mathrm{~h}$. The third axis represents only $7.6 \%$ of the total variance and con- 

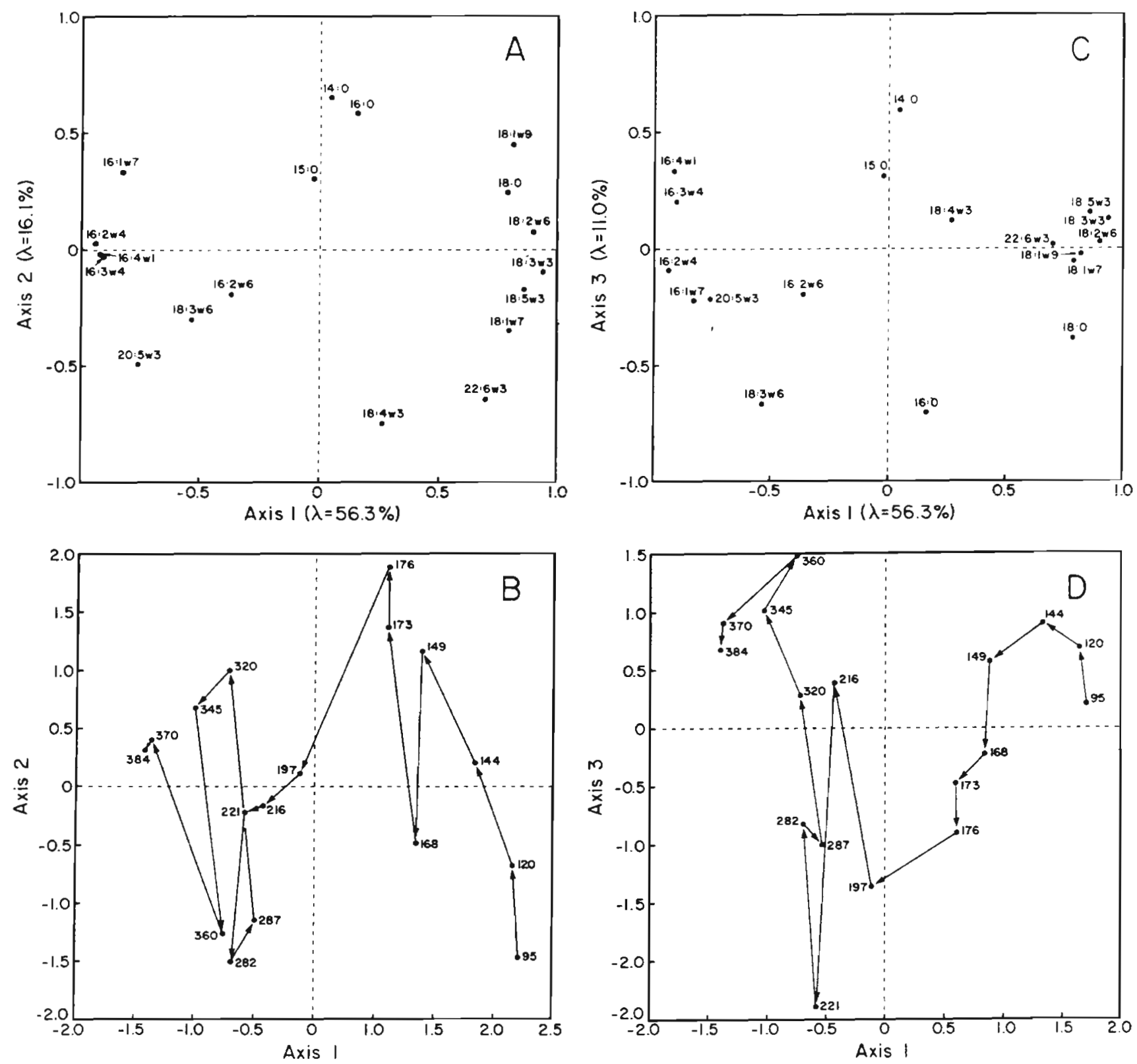

Fig. 6. Principal component analysis of the correlation matrix between total lipid fatty acids. (A) and (C) projections on the planes defined by Axes $1-2$ and $1-3$ of the correlations between variables (fatty acids) and the first 3 principal components. (B) and (D) projections of the factor scores of the observations (time of sampling in hours) illustrating the association between sampling periods. $\lambda . "$ of total variance (or inertia) explained by each axis. Arrows: time sequence of observations

trasts palmitoleic acid with 20:503 (Fig. 8C). Both axes appear to illustrate the nature of the transition and to single out 2 turning points in the pattern of changes of MGDG fatty acids. Axis 2 indicates that the influence of the initial population structure ended after $197 \mathrm{~h}$ (Fig. 8B) while Axis 3 suggests that changes in the proportion of $20: 503$ and 1610$) 7$ were characteristic of the $282 \mathrm{~h}$ sampling, i.e. the fourth growth cycle (Fig. 8D). Since that cycle was possibly limited by relatively low light intensity, this last axis may be associated to functional acclimation of the photosynthetic membranes.

Digalactosyl diglycerides and sulfoquinovosyl diglycerides (DGDG + SQDG). The fatty acid spectra for these (Table 7) are characterized by a high level of saturation with the dominance of $14: 0$ and to a minor extent 16:0. Monoenes never showed high relative concentrations and remained around 10 to $13 \%$ with $16.1 \omega 7$ as the main representative. Polyunsaturated acids reached levels equivalent to those observed for 
Table 5. Fatty acid composition of the phytoplankton neutral lipids (expressed as \% total fatty acids). A. Iso: anteiso; - . not detectable

\begin{tabular}{|c|c|c|c|c|c|c|c|c|c|c|}
\hline \multirow[t]{2}{*}{ Fatty acids } & \multicolumn{9}{|c|}{ Time (h) } & \multirow[b]{2}{*}{384} \\
\hline & 120 & 144 & 168 & 176 & 197 & 216 & 320 & 345 & 360 & \\
\hline \multicolumn{11}{|l|}{ Saturated } \\
\hline $14: 0$ & 20.59 & 22.02 & 25.84 & 18.00 & 14.89 & 20.37 & 15.85 & 20.32 & 18.30 & 18.17 \\
\hline Iso $15: 0$ & - & 0.33 & - & - & - & - & 0.21 & - & - & - \\
\hline A.Iso $15: 0$ & - & 0.16 & - & - & - & - & 0.26 & - & - & - \\
\hline $15: 0$ & 1.80 & 0.83 & 1.01 & 0.85 & 0.68 & 1.37 & 1.07 & 1.31 & 1.57 & 1.12 \\
\hline $16: 0$ & 20.74 & 14.36 & 12.96 & 16.22 & 15.31 & 13.63 & 17.21 & 12.95 & 16.00 & 11.32 \\
\hline $17: 0$ & - & 0.09 & 0.28 & - & 0.14 & - & - & - & - & 0.35 \\
\hline $18: 0$ & 3.82 & 1.27 & 1.36 & 1.51 & 1.06 & 2.02 & 1.08 & 1.12 & 1.57 & 0.83 \\
\hline $20: 0$ & - & 0.12 & 0.21 & - & - & - & 0.07 & - & - & - \\
\hline \multicolumn{11}{|l|}{ Monoenoic } \\
\hline $16: 109$ & - & 0.43 & 2.55 & - & - & - & 0.13 & - & - & 1.18 \\
\hline $16: 1 \omega^{7}$ & 12.86 & 19.43 & 19.40 & 19.21 & 22.74 & 17.66 & 23.26 & 22.31 & 19.22 & 22.78 \\
\hline $18: 1 \omega 9$ & 4.06 & 2.14 & 1.52 & 1.69 & 1.43 & 2.16 & 1.07 & 1.54 & 1.98 & 0.34 \\
\hline $18: 1 \omega 7$ & 1.59 & 0.81 & 0.84 & 0.59 & 0.91 & 1.22 & 0.51 & 0.40 & 0.43 & 0.94 \\
\hline \multicolumn{11}{|l|}{ Dienoic } \\
\hline $16: 2 \omega 6$ & 0.29 & 0.56 & 0.76 & 0.59 & 0.61 & 0.62 & - & 0.67 & 0.75 & 0.84 \\
\hline $16: 2 \omega 4$ & 1.44 & 1.70 & 1.54 & 2.21 & 2.05 & 1.99 & 1.61 & 2.17 & 2.43 & 1.84 \\
\hline $18: 2 \omega 6$ & 2.38 & 2.83 & 1.89 & 2.18 & 1.95 & 1.59 & 1.60 & 1.18 & 1.10 & 0.64 \\
\hline \multicolumn{11}{|l|}{ Trienoic } \\
\hline $16: 3 \omega 6$ & - & 0.08 & - & - & 0.05 & 0.19 & - & - & - & - \\
\hline $16: 3 \omega 4$ & 0.51 & 0.87 & 1.06 & 0.96 & 1.43 & 1.68 & 1.54 & 2.50 & 1.98 & 3.32 \\
\hline $18: 3 \omega 6$ & 0.24 & 0.31 & 0.21 & 0.23 & 0.51 & 0.35 & 0.69 & 0.31 & 0.32 & 0.42 \\
\hline $18: 3 \omega 3$ & 2.34 & 2.74 & 2.53 & 1.48 & 1.46 & 1.17 & 1.07 & 0.87 & 0.62 & 0.26 \\
\hline \multicolumn{11}{|l|}{ Tetraenoic } \\
\hline $16: 4 \omega 1$ & 1.63 & 4.32 & 4.61 & 4.50 & 4.96 & 3.19 & 4.24 & 5.46 & 5.94 & 5.51 \\
\hline $18: 4 \omega 3$ & 3.35 & 4.25 & 2.73 & 2.85 & 4.73 & 2.90 & 4.78 & 3.42 & 2.79 & 2.35 \\
\hline $20: 406$ & - & 0.28 & 0.44 & - & 0.35 & - & 0.40 & - & - & - \\
\hline $20: 4 \omega 3$ & - & 0.58 & 0.35 & 0.59 & 0.56 & - & 0.43 & - & 0.36 & - \\
\hline \multicolumn{11}{|l|}{ Pentaenoic } \\
\hline $18: 5 \omega 3$ & 2.70 & 3.40 & 2.85 & 1.83 & 1.97 & 2.04 & 1.46 & 1.11 & 0.67 & 0.30 \\
\hline $20: 503$ & 13.69 & 10.14 & 10.83 & 19.90 & 18.07 & 20.76 & 16.33 & 19.49 & 21.02 & 23.72 \\
\hline \multicolumn{11}{|l|}{ Hexaenoic } \\
\hline $22: 6 \omega 3$ & 6.64 & 5.32 & 4.39 & 4.82 & 4.14 & 5.07 & 3.91 & 2.34 & 3.02 & 3.37 \\
\hline
\end{tabular}

the neutral lipids with $18: 4 \omega 3,20: 5 \omega 3$ and $16: 2 \omega 4$ as the major constituents. The occurrence of branch-chain acids in $\mathrm{C} 15$ as well as $16: 1$ trans $\Delta 3$ seems characteristics of this fraction.

The results of the PCA analysis show that the first 3 axes explain $87 \%$ of the total variance. The first axis accounts for $46.8 \%$ of the total inertia and opposes the saturated acids in C14 and C16 to the C16 PUFA and 20:5w3 (Fig. 9A). It singles out those periods characteristic of the final growth cycles in opposition to the early phase of the first cycle $(120 \mathrm{~h})$ and the transition phase between the 2 systems (197 h) (Fig. 9B). The second axis explains $28.7 \%$ of the variance and seems characteristic of the C18 monoenes and polyunsaturates (Fig. 9A). It appears to be representative of the processes through which the succession from one population to the other occurred (Fig. 9B). The factorial plane defined by the first 2 axes clearly illustrates that the selective pressure reached its maximum during the second growth cycle $(197 \mathrm{~h})$ and took place through a transient resaturation of the DGDG fraction. The third axis only accounts for $11.6 \%$ of the inertia. It represents the adaptative changes which took place during the first and to a minor extent second growth cycle (120 to $168 \mathrm{~h}$ and 176 to $216 \mathrm{~h}$ ) which are associated to the decrease in the relative proportion of $18: 403$ and the increase in 15:0,16:0 and 18:0 (Fig. 9C, D). As indicated by the projection in the factorial plane $2-3$, it appears that the oscillations in the DGDG structure produced by the external changes are damped as the system proceeded through time towards a new equilibrium (Fig. 9D).

Phospholipids (PL). The fatty acid composition of PL fraction is given in Table 8 and displays a high degree of unsaturation with the dominance of $20: 5 \omega 3$ and/or $22: 6 \omega 3$. The percentage of monoenes varied between 20 and $34 \%$ with $16: 1 \omega 7$ as the major compound. The general dominance of $18: 1 \omega 7$ over $18: 1 \omega 9$ is remarkable and suggests an active elongation process of the palmitoleic acid which decreased over time. The satu- 

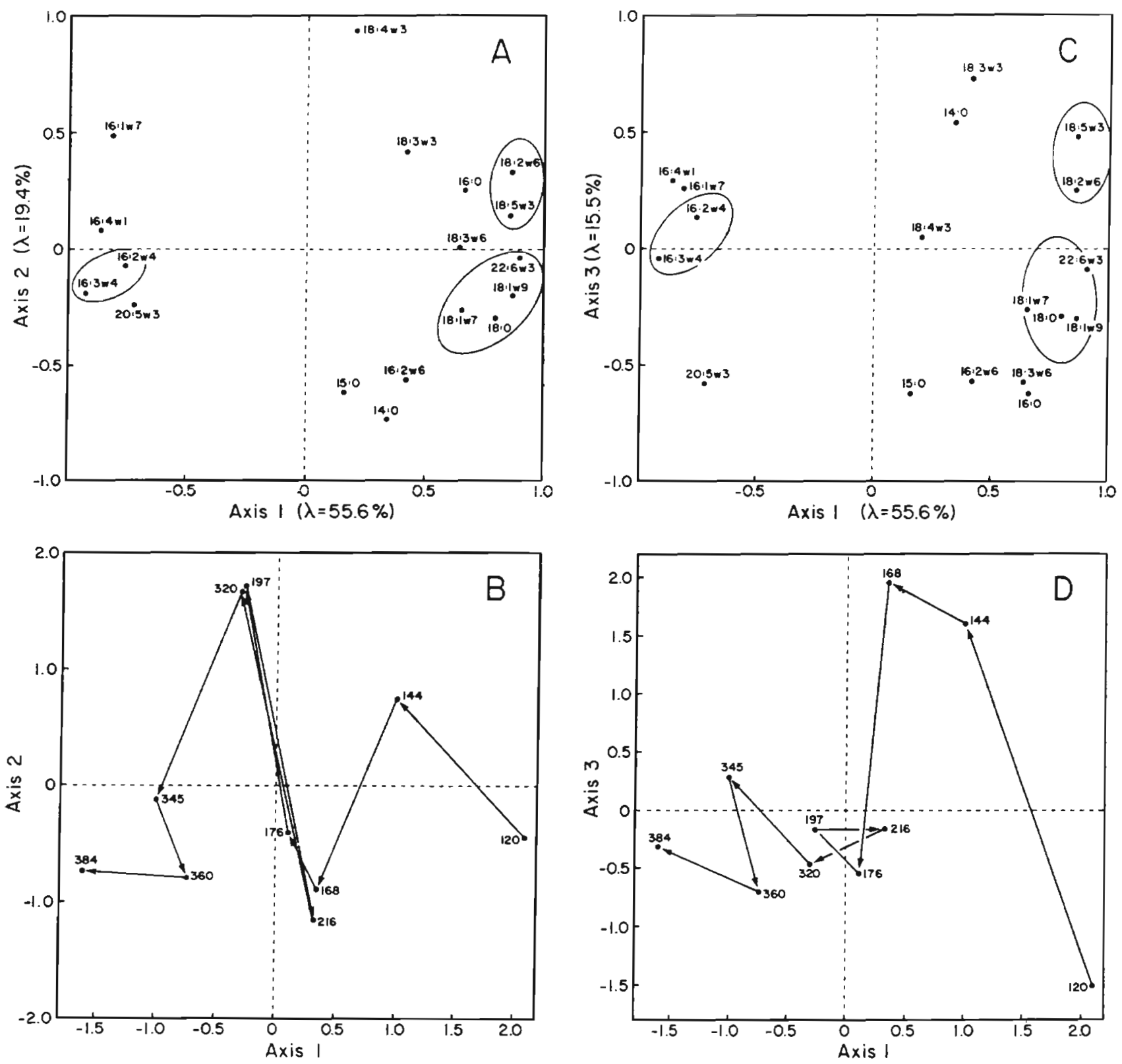

Fig. 7. Principal component analysis of the correlation matrix between neutral lipid fatty-acids. (A) and (C) Factor loadings on the first 3 axes illustrating the correlations of the variables with the first 3 principal components. (B) and (D) Factor scores illustrating the association between sampling periods and the corresponding time sequence. Symbols as in Fig. 6

rated acids included large proportions of $\mathrm{C} 16: 0$ and C14:0 as well as the occurrence of branch-chain acids in $\mathrm{C} 15$.

From the PCA analysis, it can be shown that the first 3 factorial axes account for $88.2 \%$ of the total variance The first one explains $50.4 \%$ of the inertia and contrasts $20: 5(1) 3,16: 1(1) 7$ and $16: 3(1) 4$ with $18 \cdot 107$, 18:303 and 18:503 (Fig. 10A). As expected, it illustrates the replacement of the initial complex phytoplankton population (120 to $168 \mathrm{~h}$ ) by a diatom-dominated one (216 to $384 \mathrm{~h}$ ) (Fig. 10B). The second axis accounts for $26.9 \%$ of the total variance and is defined by the saturates $(16: 0,18: 0,15: 0)$ and oleic acid on the one hand and C18 PUFA $(18: 5 \omega 3,18: 3 \omega 3,18: 4(13)$ and $22: 603$ on the other hand (Fig. 10A). As suggested earlier for DGDG, it appears to be representative of the processes of resaturation which characterize the transition between the 2 populations during the second growth cycle (197 h) (Fig. 10B). The third axis explains $10.9 \%$ of the total variance and opposes $14: 0$ and $16: 2 \omega 6$ to $18: 2 \omega 6$ (Fig. 10C). It singles out 2 sampling times, one at the end of the first growth cycle (168 h) 
Table 6. Fatty acid composition of the phytoplankton monogalactosyl diglycerides (MGDG) (expressed as \% total fatty acids). - Not detected

\begin{tabular}{|c|c|c|c|c|c|c|c|c|c|c|c|}
\hline \multirow[t]{2}{*}{ Fatty acids } & \multicolumn{9}{|c|}{ Time (h) } & \multirow[b]{2}{*}{360} & \multirow[b]{2}{*}{384} \\
\hline & 120 & 144 & 168 & 176 & 197 & 216 & 282 & 320 & 345 & & \\
\hline \multicolumn{12}{|l|}{ Saturated } \\
\hline $14: 0$ & 16.11 & 13.81 & 13.00 & 13.52 & 8.80 & 8.85 & 5.52 & 9.08 & 7.00 & 6.48 & 3.24 \\
\hline $15: 0$ & 1.36 & 1.17 & 1.94 & 0.71 & 0.73 & 0.91 & 0.45 & 0.55 & 0.57 & 0.55 & 0.51 \\
\hline $16: 0$ & 5.74 & 9.80 & 13.22 & 8.98 & 12.42 & 7.04 & 4.86 & 6.10 & 4.71 & 3.85 & 4.81 \\
\hline $18: 0$ & 0.46 & 1.20 & 1.98 & 0.71 & 1.15 & 1.08 & 1.00 & 0.19 & 0.20 & 0.24 & 0.25 \\
\hline $20: 0$ & - & 0.17 & 0.55 & - & - & - & - & - & - & - & - \\
\hline \multicolumn{12}{|l|}{ Monoenoic } \\
\hline $16: 1 \omega 9$ & 0.08 & 0.92 & 0.24 & - & - & - & - & 0.13 & 0.09 & - & - \\
\hline $16: 107$ & 6.02 & 9.34 & 9.38 & 13.02 & 11.89 & 10.18 & 7.65 & 12.69 & 13.75 & 8.70 & 11.66 \\
\hline $18: 1 \omega 9$ & 2.07 & 1.91 & 2.52 & 1.46 & 1.06 & 0.89 & 0.74 & 0.76 & 0.37 & 0.55 & 0.43 \\
\hline $18: 107$ & 0.67 & 0.52 & 0.52 & 0.46 & 0.61 & 0.36 & 0.22 & 0.23 & 0.28 & 0.11 & 0.25 \\
\hline \multicolumn{12}{|l|}{ Dienoic } \\
\hline $16: 2 \omega 6$ & 0.38 & 0.50 & 0.66 & 0.82 & 0.75 & 1.04 & 1.44 & 1.04 & 1.36 & 1.77 & 1.70 \\
\hline $16: 2 \omega 4$ & 1.36 & 1.77 & 2.32 & 2.62 & 2.31 & 3.90 & 4.29 & 2.80 & 3.90 & 3.64 & 4.08 \\
\hline $18: 2 \omega 6$ & 2.93 & 2.63 & 1.59 & 2.25 & 1.29 & 0.63 & 1.10 & 1.18 & 0.50 & 0.54 & 0.30 \\
\hline \multicolumn{12}{|l|}{ Trienoic } \\
\hline $16: 3 \omega 4$ & 2.53 & 3.51 & 4.71 & 5.89 & 6.09 & 12.53 & 13.52 & 9.24 & 12.61 & 11.41 & 16.65 \\
\hline $16: 3 \omega 6$ & 0.31 & 0.21 & 0.30 & 0.36 & 0.33 & 0.29 & 0.27 & 0.19 & 0.28 & 0.24 & 0.29 \\
\hline $18: 3 \omega 3$ & 1.98 & 1.77 & 1.05 & 1.02 & 0.65 & 0.31 & 0.21 & 0.32 & 0.25 & 0.18 & 0.21 \\
\hline \multicolumn{12}{|l|}{ Tetraenoic } \\
\hline $16: 101$ & 8.03 & 9.16 & 11.09 & 16.03 & 15.84 & 23.92 & 27.44 & 27.88 & 31.43 & 41.82 & 30.98 \\
\hline $18: 4 \omega 3$ & 11.45 & 6.17 & 6.60 & 6.92 & 8.47 & 5.82 & 5.84 & 6.46 & 5.71 & 4.01 & 4.84 \\
\hline \multicolumn{12}{|l|}{ Pentaenoic } \\
\hline $18: 5 w 3$ & 27.72 & 20.77 & 13.22 & 10.18 & 5.42 & 2.48 & 2.73 & 4.57 & 2.46 & 2.28 & 0.83 \\
\hline $20: 5 w 3$ & 7.46 & 9.34 & 12.47 & 14.18 & 21.03 & 19.08 & 21.62 & 15.55 & 14.17 & 13.04 & 19.10 \\
\hline \multicolumn{12}{|l|}{ Hexaenoic } \\
\hline $22: 6 \omega 3$ & 1.54 & 2.25 & 2.93 & 1.42 & 1.16 & 0.70 & 0.55 & 0.52 & 0.48 & 0.28 & 0.10 \\
\hline
\end{tabular}

and one at the beginning of the fifth cycle $(320 \mathrm{~h})$ both characterized by relatively high percentages of saturated acids (Fig. 10D). It is likely that such specific behaviour is related to cellular changes occurring before and after dilutions since similar changes were noted with the neutral lipid fraction.

\section{DISCUSSION}

Although the study was designed to observe successive growth cycles regulated by nutrient depletion, proper interpretation of the changes recorded must consider 3 experimental constraints: (1) a natural body of water with its complex particle assemblage was trapped, (2) the medium was enriched with nutrients at levels far higher than any natural levels, and (3) the system was subjected to natural changes in light and temperature. Thus, our system should be viewed as a large-scale culture of a natural phytoplankton population subjected to natural physical variations and simulated nutrient pulses.

The different growth cycles illustrate well such characteristics. The first one showed the longest dura- tion, i.e. $174 \mathrm{~h}(7.3 \mathrm{~d})$, the slowest nutrient uptake and the smallest protein exponential growth rates. Levels of biomass were low and showed a definite detrital component. Changes in particulate volume, chlorophyll, protein and lipids showed an exponential increase, though per unit volume both protein and lipids appeared to be sensitive to nutrient reduction and decrease with increasing limitation. In terms of total fatty acids, inverse variations of $\mathrm{C} 16$ and C18 PUFA, associated with the progressive dominance of diatoms, are the major characteristic. The other 5 growth cycles were of much shorter duration, i.e. 27 to $51 \mathrm{~h}$, displayed faster nutrient uptake and increasing exponential growth rates. The lower growth rate during Cycle 4 was likely related to lower light regime around Day 10 as light saturation for Skeletonema costatum requires light intensities greater than $144 \mathrm{cal} \mathrm{m}^{-2} \mathrm{~d}^{-1}$ (Augier unpubl.). The higher growth rates seemed associated with larger dilution rates. Phytoplankton and mainly diatoms dominated all 5 cycles and, except during the fourth one, reached levels of biomass almost 3 times higher than during the first cycle. A decrease of protein and lipid contents per unit cell volume with nutrient reduction seems to be a feature common to all cycles. 

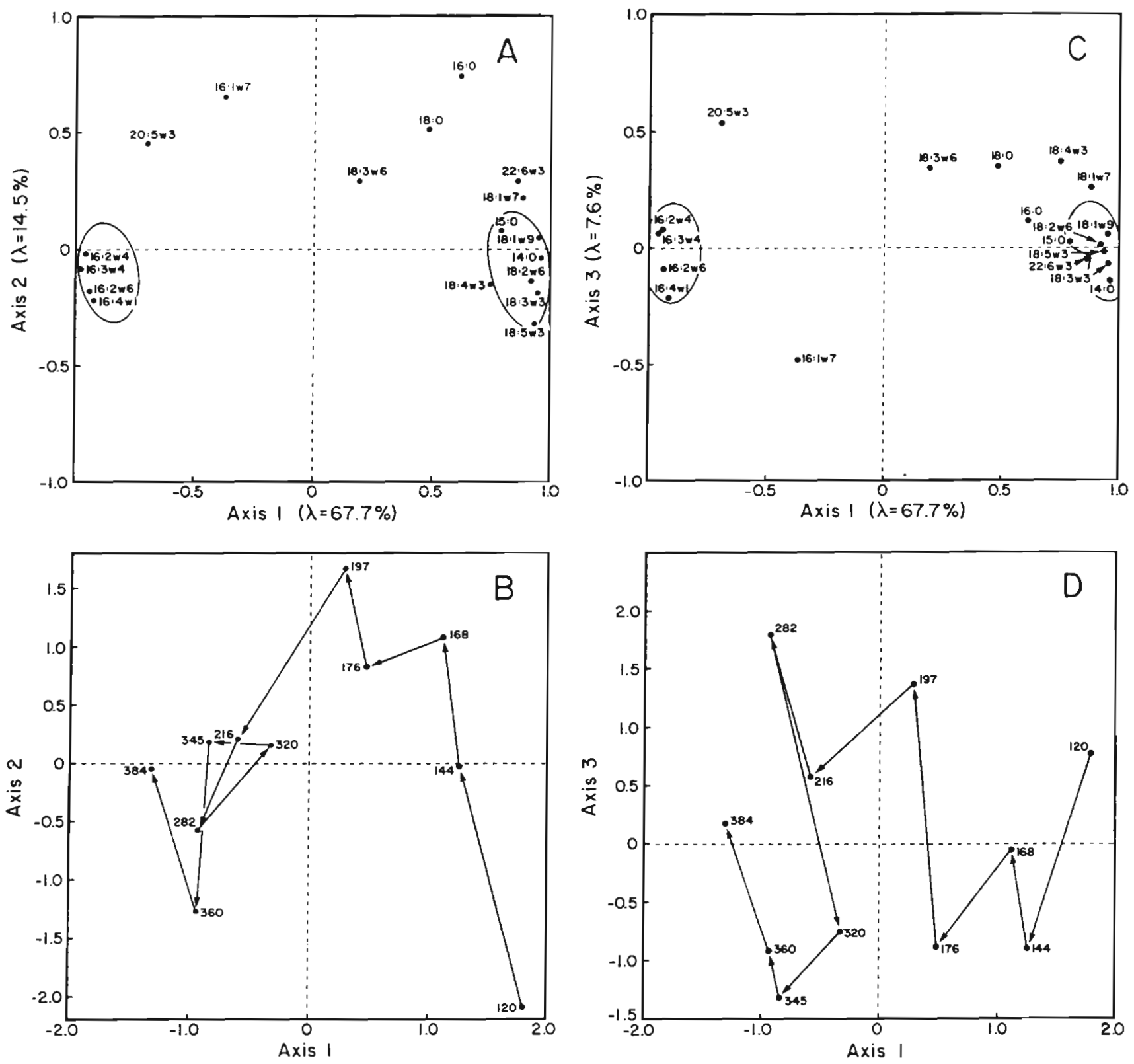

Fig. 8. Principal component analysis of the correlation matrix between monogalactosyl diglycericles (MGDG) fatty acids. (A) and (C) Factor loadings on the first 3 axes, illustrating the correlations between fatty acids and the first 3 principal components. (B) and

(D) Factor scores illustrating the association between sampling time and the corresponding sequence. Symbols as in Fig. 6

Changes in total fatty acids indicated that species selection was finalized during the second cycle with increasing relative concentration of the C16 PUFA which largely dominated during the last 2 cycles. Over the entire time sequence, changes in cell global biochemistry appear to have resulted from selective pressure and cell acclimation to high nutrient levels which produced high yields of biomass. Because our experiment was designed to prevent severe mutrient limitation, reduction of protein concentration or enhanced lipid accumulation usually observed during stationary growth phases or towards the end of natural blooms (Figg 1956, Badour \& Gergis 1965, Haug et al. 1973, Morris et al. 1983, 1985, Parrish 1987, Mayzaud et al. 1989) were not observed.

Different studies have dealt with changes in component fatty acids of phytoplankton during natural or experimentally produced blooms (Kattner et al. 1983, Morris 1984, Morris et al. 1985, Mayzaud et al. 1989) but none have considered the influence of variable nutrient regime. Under natural conditions, fatty acid composition was found to be related to species succes- 
Table 7. Fatty acid composition of the phytoplankton digalactosyl diglycerides (DGDG) and sulfoquinovosyl diglycerides (SQDG) (expressed as \% total fatty acids). A.Iso: anteiso; - . not detected

\begin{tabular}{|c|c|c|c|c|c|c|c|c|c|c|c|}
\hline \multirow[t]{2}{*}{ Fatty acids } & \multicolumn{11}{|c|}{ Time (h) } \\
\hline & 120 & 144 & 168 & 176 & 197 & 216 & 282 & 320 & 345 & 360 & 384 \\
\hline \multicolumn{12}{|l|}{ Saturated } \\
\hline $14: 0$ & 35.34 & 25.67 & 18.86 & 36.17 & 46.73 & 25.76 & 27.82 & 24.32 & 34.35 & 32.76 & 35.02 \\
\hline Iso 15:0 & 0.37 & 0.47 & 0.21 & 0.43 & 0.18 & 0.21 & 0.37 & 0.36 & 0.47 & 0.23 & 0.18 \\
\hline A.Iso $15: 0$ & 0.20 & 0.49 & 0.23 & 0.38 & 0.34 & 0.51 & 0.79 & 0.18 & 0.19 & 0.25 & 0.31 \\
\hline $15: 0$ & 0.84 & 1.39 & 1.28 & 0.77 & 1.80 & 1.24 & 1.12 & 1.42 & 1.11 & 1.16 & 1.26 \\
\hline $16: 0$ & 11.61 & 14.42 & 13.91 & 15.36 & 29.35 & 18.21 & 13.01 & 14.22 & 11.25 & 14.31 & 9.09 \\
\hline $18: 0$ & 1.00 & 1.55 & 2.11 & 0.51 & 1.53 & 1.01 & 1.06 & 1.28 & 0.66 & 1.85 & 0.53 \\
\hline \multicolumn{12}{|l|}{ Monoenoic } \\
\hline $16: 1 \omega 9$ & - & 1.18 & 0.40 & - & 2.40 & - & 0.18 & 2.04 & - & - & 1.18 \\
\hline $16: 1 \omega 7$ & 6.65 & 9.87 & 9.85 & 11.84 & 7.54 & 11.62 & 12.44 & 12.69 & 12.64 & 8.89 & 11.40 \\
\hline $16: 1 \operatorname{trans} \triangle 3$ & Tr & $\operatorname{Tr}$ & $\operatorname{Tr}$ & $\operatorname{Tr}$ & Tr & 0.31 & 0.27 & 0.40 & 0.36 & 0.60 & 0.18 \\
\hline $18: 1009$ & 1.49 & 1.61 & 1.81 & 0.76 & 1.00 & 0.52 & 0.65 & 2.17 & 0.65 & 0.45 & 0.39 \\
\hline $18: 167$ & 0.28 & 0.53 & 0.69 & 0.59 & 0.16 & 0.77 & 0.80 & 0.66 & 0.65 & 0.77 & 0.88 \\
\hline \multicolumn{12}{|l|}{ Dienoic } \\
\hline $16: 206$ & 0.28 & 0.53 & 0.69 & 0.59 & 0.16 & 0.77 & 0.80 & 0.66 & 0.65 & 0.77 & 0.88 \\
\hline $16: 2 \omega 4$ & 2.13 & 4.69 & 5.11 & 4.67 & 1.96 & 4.44 & 5.97 & 6.71 & 6.18 & 5.48 & 5.09 \\
\hline $18: 2 \omega 6$ & 2.01 & 1.81 & 1.59 & 1.05 & 0.47 & 0.45 & 0.44 & 1.42 & 0.47 & 0.32 & 0.19 \\
\hline \multicolumn{12}{|l|}{ Trienoic } \\
\hline $16: 3 \omega 6$ & - & 0.17 & 0.23 & - & 0.05 & - & -- & - & 0.09 & - & - \\
\hline $16: 304$ & 0.51 & 0.71 & 3.10 & 0.61 & 0.22 & 1.37 & 1.39 & 1.40 & 1.08 & 0.92 & 1.72 \\
\hline $18: 3 \omega 6$ & 0.35 & 0.30 & 0.30 & 0.33 & 0.30 & 0.50 & 0.45 & 0.39 & 0.40 & 0.27 & 0.08 \\
\hline $18: 3 \omega 3$ & 6.45 & 3.34 & 2.01 & 1.51 & 0.12 & 0.73 & 0.40 & 1.03 & 0.46 & 0.38 & 0.15 \\
\hline \multicolumn{12}{|l|}{ Tetraenoic } \\
\hline $16: 4 \omega 1$ & 1.25 & 2.98 & 8.44 & 1.67 & 0.42 & 7.61 & 4.63 & 6.15 & 3.98 & 4.39 & 5.92 \\
\hline $18: 4 \omega 3$ & 12.60 & 11.00 & 10.60 & 9.66 & 2.36 & 14.83 & 13.75 & 10.40 & 14.23 & 15.73 & 16.27 \\
\hline $20: 4 \omega 6$ & - & 0.18 & - & - & 0.35 & - & 1.36 & 0.09 & - & - & - \\
\hline $20: 4 \omega 3$ & - & 0.11 & - & - & 0.36 & - & 0.15 & 0.22 & - & - & - \\
\hline \multicolumn{12}{|l|}{ Pentaenoic } \\
\hline $18: 503$ & 6.10 & 7.45 & 8.21 & 2.19 & 0.37 & 1.11 & 1.02 & 2.32 & 1.35 & 0.83 & 0.32 \\
\hline $20: 5 \omega 3$ & 4.76 & 6.60 & 10.82 & 8.41 & 2.20 & 11.66 & 8.70 & 7.71 & 9.19 & 8.41 & 9.10 \\
\hline \multicolumn{12}{|l|}{ Hexaenoic } \\
\hline $22: 603$ & 0.84 & 1.39 & 1.28 & 0.77 & 1.80 & 1.24 & 1.12 & 1.42 & 1.11 & 1.16 & 1.26 \\
\hline Tr: trace & & & & & & & & & & & \\
\hline
\end{tabular}

sion and phytoplankton physiological state (Jeffries 1970, Kattner et al. 1983, Mayzaud et al. 1989) while it seemed to illustrate the influence of growth conditions, nutrient status and growth stage under mesocosm conditions (Morris 1984, Morris et al. 1985). Because of the initial natural particle assemblage, our results illustrate an intermediate situation. Partition of the total variance of fatty acid changes by principal component analysis showed that the major fraction was primarily related to species selection during the transition toward a diatomdominated population while nutrient oscillations and physiological changes at the time of the species transition usually accounted for less than $40 \%$ of the variance associated with the second and third principal component.

Consideration of the fatty acid changes per lipid classes rather than total lipids gives some insights as to the physiological processes involved. A recent report by Palmisano et al. (1988) has shown that under natural bloom conditions, diatoms profoundly altered their rate of synthesis of neutral lipids, and to a minor extent glycolipids, in response to successive growth phases. Distinctive patterns of fatty acid for each class have been illustrated for both cultured phytoplankton (cf. review by Pöhl \& Zurheide 1979, Holz 1981) and in situ simulated blooms (Morris 1984, Morris et al. 1985). All concur that neutral acyl glycerols are more saturated than the polar lipids with the dominance of $16: 0,16: 1$, $18: 0,14: 0$ and $18: 1$, and that both glycolipids and phospholipids include high levels of C16, C20 or C18 and C22 polyunsaturated acids. MGDG seems to be the most unsaturated lipid class, followed by phospholipids, DGDG and SQDG. Depending on the class of microalgae considered (flagellates or diatoms) either C16 PUFA and 20:5 or C18 PUFA and 22:6 will dominate (Nichols 1965, Bloch et al. 1967, Nichols et al. 

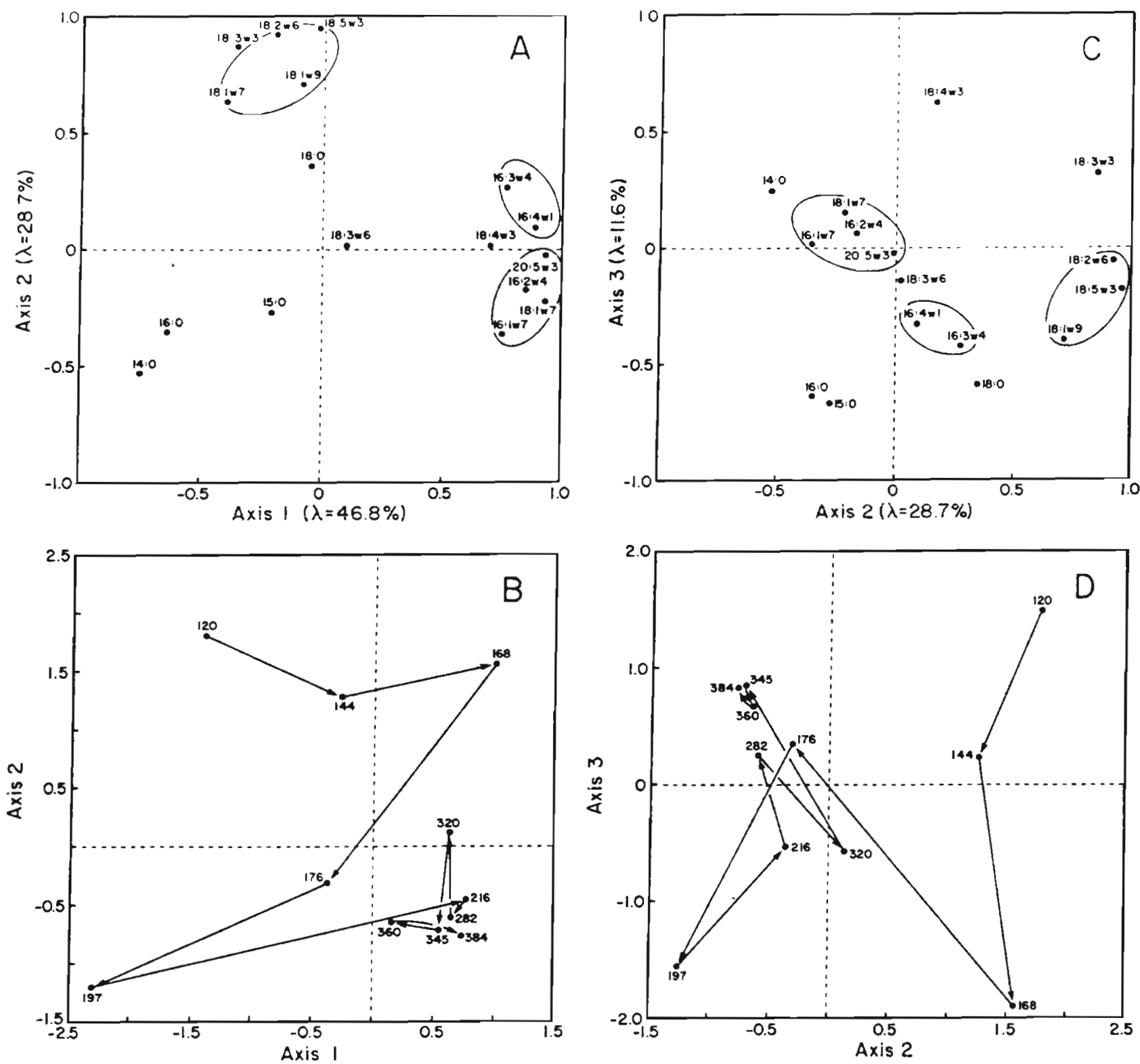

Fig. 9. Principal component analysis of the correlation matrix between digalactosyl diglycerides (DGDG) and sulfoguivosyl diglycerides (SQDG) fatty acids. (A) and (C) Factor loadings on the first 3 axes grouping those fatty acids with similar correlation levels with the principal components. (B) and (D) Factor scores of the observations illustrating the association between sampling time and the corresponding sequence. Symbols as in Fig. 6

1967, Pöhl \& Zurheide 1979, Holz 1981, Arao et al 1987, present study).

Because differences in structure are often the result of differences in metabolic pathways, it is interesting to consider in some detail the influence of the successive growth cycles on the fatty acid composition of each lipid class as can be extrapolated from the PCA analysis. The transition from a flagellate to a diatom population appears to be a key factor influencing all categories in a similar way, i.e, transition from a [C18 PUFA, 22:6w3] pattern to a [C16 PUFA, 20:5(1)3, $\left.18: 1(1) 9,18: 1 \omega^{7}\right)$ pattern. Once this source of variance is extracted, the results of the analysis indicate that the same set of external parameters produced different sequences of fatty acid changes for the different lipid classes.

If the physiological interpretation of the principal components is valid, it would appear that the nutrient regime affected the neutral lipids essentially through a process of saturation-desaturation which was best illustrated by the time changes of $14: 0,15: 0,16: 206$ before dilution and 18:4(1)3 after dilution. The changes 
Table 8. Fatty acid composition of the phytoplankton phospholipids (expressed as \% total fatty acids). A. Iso: anteiso; - not detected

\begin{tabular}{|c|c|c|c|c|c|c|c|c|c|c|c|c|}
\hline \multirow[t]{2}{*}{ Fatty acids } & \multicolumn{9}{|c|}{ Time (h) } & \multirow[b]{2}{*}{360} & \multirow[b]{2}{*}{370} & \multirow[b]{2}{*}{384} \\
\hline & 120 & 144 & 168 & 176 & 197 & 216 & 282 & 320 & 345 & & & \\
\hline \multicolumn{13}{|l|}{ Saturated } \\
\hline $14: 0$ & 8.41 & 7.85 & 14.66 & 7.83 & 10.89 & 7.30 & 9.09 & 14.40 & 7.35 & 6.63 & 6.27 & 6.13 \\
\hline Iso $15: 0$ & 0.45 & 0.68 & 0.70 & 0.82 & 0.81 & 0.99 & 0.77 & 0.63 & 0.25 & 0.93 & 0.26 & 0.59 \\
\hline A. Iso $15: 0$ & 0.19 & 0.37 & 0.38 & 0.41 & 0.47 & 1.02 & 0.94 & 0.70 & 0.30 & 0.31 & 0.26 & 0.30 \\
\hline $15: 0$ & 0.82 & 1.17 & 1.27 & 0.93 & 2.76 & 1.85 & 1.05 & 0.76 & 0.58 & 0.62 & 0.71 & 0.08 \\
\hline $16: 0$ & 12.13 & 14.63 & 14.66 & 12.80 & 20.26 & 12.18 & 12.29 & 13.00 & 12.35 & 9.89 & 12.23 & 12.62 \\
\hline $17: 0$ & - & 0.23 & 0.19 & - & - & - & 0.15 & 0.09 & - & - & 0.12 & 0.43 \\
\hline $18: 0$ & 0.76 & 1.26 & 1.05 & 0.73 & 2.67 & 1.05 & 0.82 & 0.40 & 0.45 & 0.50 & 0.55 & 0.61 \\
\hline $20: 0$ & - & 0.09 & 0.14 & - & - & - & - & - & 0.15 & - & - & - \\
\hline \multicolumn{13}{|l|}{ Monoenoic } \\
\hline $16: 1 \omega 9$ & - & - & - & 0.09 & 4.10 & - & 0.28 & - & - & - & - & - \\
\hline $16: 1 \omega 7$ & 10.18 & 14.11 & 16.30 & 16.19 & 19.78 & 20.13 & 19.26 & 18.98 & 25.18 & 23.84 & 24.92 & 26.50 \\
\hline $17: 1010$ & - & 0.12 & - & - & - & - & 0.07 & 0.09 & - & - & 0.32 & - \\
\hline $18: 109$ & 1.76 & 1.42 & 2.09 & 1.17 & 4.65 & 0.66 & 0.94 & 0.63 & 0.43 & 0.65 & 0.48 & 0.86 \\
\hline $18: 107$ & 9.02 & 7.32 & 6.46 & 5.93 & 5.73 & 5.09 & 3.57 & 2.81 & 1.58 & 1.56 & 1.25 & 1.40 \\
\hline \multicolumn{13}{|l|}{ Dienoic } \\
\hline $16: 2 \omega 6$ & 0.47 & 0.42 & 0.46 & 0.39 & 0.26 & 0.61 & 0.59 & 1.27 & 0.38 & 0.67 & 0.52 & 0.82 \\
\hline $16: 204$ & 0.70 & 0.81 & 1.25 & 1.28 & 1.85 & 1.72 & 1.56 & 0.60 & 1.22 & 1.60 & 2.13 & 1.16 \\
\hline $18: 2 \omega 6$ & 1.34 & 1.34 & 1.10 & 1.49 & 1.25 & 1.22 & 0.84 & 1.02 & 1.40 & 0.90 & 1.20 & 1.06 \\
\hline \multicolumn{13}{|l|}{ Trienoic } \\
\hline $16: 3 \omega 6$ & - & 0.09 & 0.18 & 0.07 & - & - & - & - & -- & 0.07 & - & 0.12 \\
\hline $16: 304$ & 0.35 & 0.47 & 0.93 & 0.94 & 0.01 & 0.98 & 1.07 & 1.42 & 0.87 & 1.21 & 2.05 & 1.26 \\
\hline $18: 3 \omega 6$ & 0.55 & 0.39 & 0.36 & 0.60 & 0.31 & 0.65 & 0.52 & 0.53 & 0.52 & 0.49 & 0.46 & 0.67 \\
\hline $18: 3 \omega 3$ & 1.71 & 1.18 & 1.05 & 0.85 & 0.34 & 0.52 & 0.37 & 0.55 & 0.36 & 0.26 & 0.32 & 0.18 \\
\hline \multicolumn{13}{|l|}{ Tetraenoic } \\
\hline $16: 4 \omega 1$ & 0.35 & 1.39 & 1.49 & 2.06 & 1.51 & 1.33 & 1.40 & 1.42 & 1.79 & 3.42 & 5.48 & 4.72 \\
\hline $18: 4 \omega 3$ & 5.30 & 3.34 & 4.75 & 3.99 & 2.54 & 4.50 & 5.41 & 6.35 & 5.70 & 6.65 & 0.29 & 0.59 \\
\hline $20: 4 \omega 6$ & - & 0.42 & - & - & - & - & - & 0.22 & - & - & 0.34 & 0.43 \\
\hline $20: 4 \omega 3$ & - & 0.55 & 0.35 & - & - & - & 0.35 & 0.30 & 0.31 & 0.54 & 0.59 & 0.20 \\
\hline \multicolumn{13}{|l|}{ Pentaenoic } \\
\hline $18: 5(1) 3$ & 2.84 & 2.46 & 1.31 & 1.72 & 0.39 & 0.44 & 0.26 & 0.73 & 0.50 & 0.54 & 0.59 & 0.20 \\
\hline $20: 5(03$ & 1780 & 16.90 & 15.93 & 22.61 & 16.14 & 28.42 & 24.96 & 21.98 & 27.96 & 28.32 & 25.98 & 23.01 \\
\hline \multicolumn{13}{|l|}{ Hexaenoic } \\
\hline $22: 6 w 3$ & 22.52 & 18.40 & 13.28 & 16.65 & 6.94 & 11.36 & 12.67 & 11.98 & 11.03 & 11.52 & 11.01 & 13.98 \\
\hline
\end{tabular}

in physiological state during the first and part of the second growth cycle, related to the shift in phytoplankton community structure (i.e. selective pressure), affected to a varying extent the different lipid classes. Unrecorded for the neutral lipid fatty acids, they are second in importance (Axis 2) for MGDG, DGDG, SQDG and phospholipids and suggest the occurrence of a transient resaturation of the membrane fatty acids at the onset of the diatom population with C18 PUFA characteristic descriptors being replaced by more saturated acids $(16: 0,18: 0,15: 0,16: 1 \omega 7,18: 1 \omega 9)$.

Adaptation of membrane fatty acid constituents to growth-limiting light intensity has been little studied in marine phytoplankton species. Recently, Mortensen et al. (1988) reported little effect on the total fatty acid composition of the diatom Chaetoceros gracilis. They described increased levels of highly unsaturated acids ( $\omega 3$ or $n-3$ ) with increasing light intensity but with hardly any variation of the unsaturated to saturated acids ratio and polyunsaturated to monoenoic plus saturated acids ratio. In an earlier study, based on fatty acid changes of lipid classes (polar, diglycerides, triglycerides, free fatty acids), Orcutt \& Patterson (1974) found an increase in polar lipid fatty acids and a decrease in neutral lipid fatty acids at low light intensity for the diatom Nitzschia closterium. Depending on the lipid class considered, the major changes were recorded for $16: 3+18: 1,20: 5,16: 1$ and $16: 0$. In the present study, principal component analysis singled out the possibly light-limited fourth cycle only for

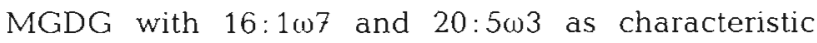
descriptors. Lower light intensity seemed to increase the relative proportion of $20: 5$ and decrease the level of 16:1 without corresponding changes of the $16: 3$ or 16:4 and 16:0 percentages. Such a conclusion cannot be directly compared with earlier results as the present ones do not derive from experimental situations where light is the only forcing function but agree with the 

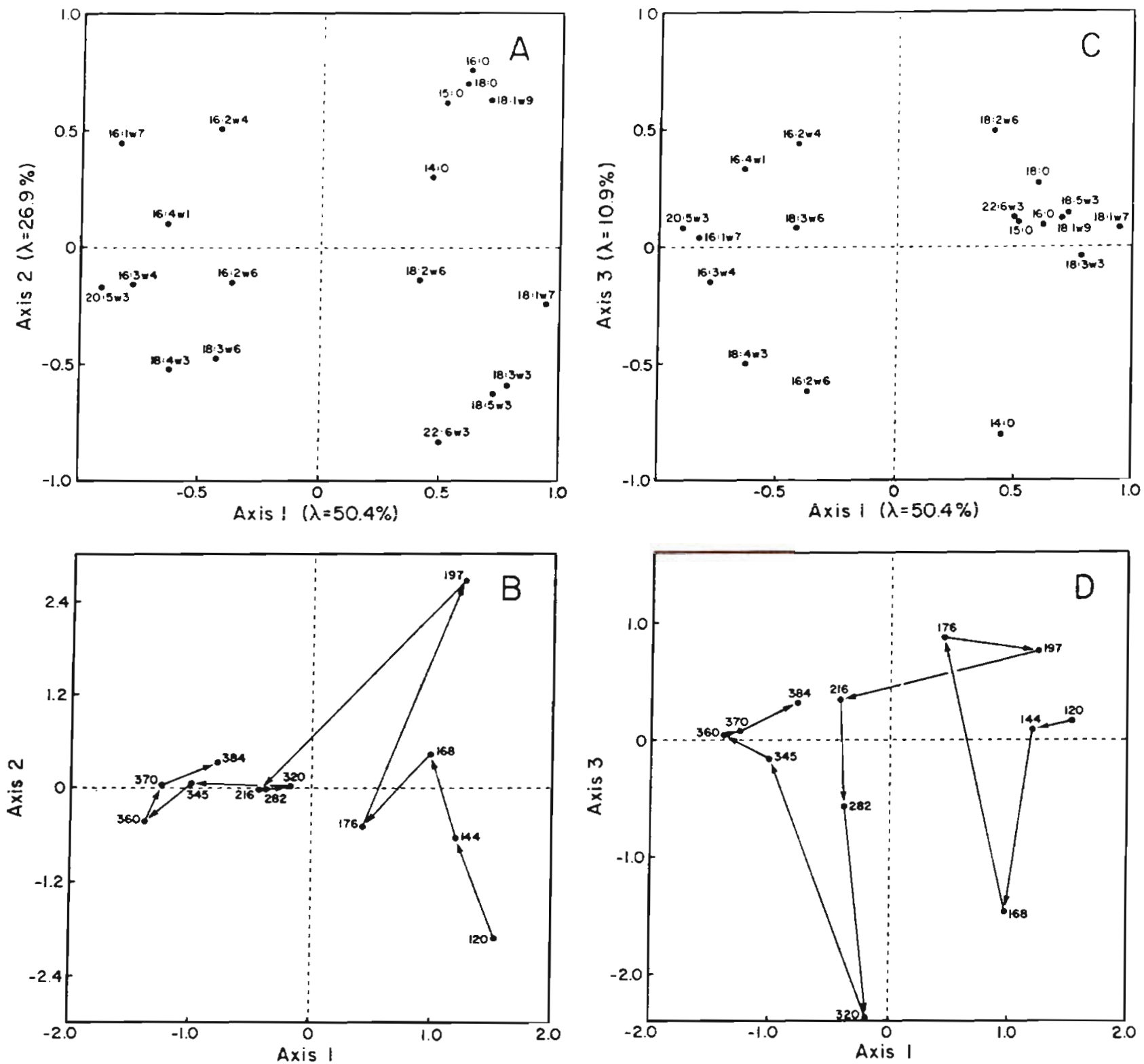

Fig. 10. Principal component analysis of the correlation matrix between phospholipid (PL) fatty acids. (A) and (C) Factor loadings on the first 3 axes, illustrating the correlations between fatty acids and the principal components. (B) and (D) Factor scores of the

observations, illustrating the association between sampling time and the corresponding sequence. Symbols as in Fig. 6

general findings that light regime affects essentially membrane chloroplast lipid through changes in the level of 13 polyunsaturated fatty acids.

The role of long-chain polyunsaturated acids in phytoplankton physiology remains unclear. Morris et al. (1985) pointed out the discrepancy between the high levels recorded in most cultured species and natural populations under bloom conditions. Indeed, while most cultured diatoms and dinoflagellates displayed levels of $20: 5 w 3$ and/or $22: 6 w 3$ between 7 and $30 \%$ of the total pool of fatty acids (Pöhl \& Zurheide 1979), the data reported by Kattner et al. (1983) and Mayzaud et al.
(1989) for natural spring blooms or by Morris (1984) and Morris et al. (1985) for experimental blooms fell in the range 0 to $10 \%$ of total fatty acids. Morris et al. (1985) associated this difference with the fast growth rate of their population which either might not have the time to synthesize the more complex long-chain polyunsaturated components or displayed specific requirements for variable cellular membrane fluidity to cope with a changing nutrient regime. The data reported in the present study bring some evidence in support of the second hypothesis since high percentages of both $20: 5 \omega 3$ and $22: 603$ were found in all lipid classes 
despite quite variable growth rates, different duration of growth cycle ( 1 to $7 \mathrm{~d}$ ), and nutrient pulses. Adaptative acclimation to species selection, light limitation, and growth phases appeared to proceed through transient changes in the PUFA of polar lipids which are known to regulate membrane fluidity. The known association between relative concentration of PUFA and nutrient regime (Pöhl \& Zurbeide 1979, Shifrin \& Chisholm 1981, Enright et al. 1986, Mortensen et al. 1988) suggests some functional relationship between membrane fluidity and nutrient availability which in most culture systems is obscured during the stationary growth phase. In the present experiment, the maintenance throughout the different growth cycles of exponential growth strongly suggests that in culture or culture-like system high levels of PUFA could be related to permeability requirements to cope with the high nutrient situation.

Acknowledgements. We thank Dr P. Nival for his continuous support and the use of tank facilities. Very valuable assistance during the course of the study were provided by Mrs C. Cellario, Mr G. Malara and Mr R. Charra. This work was supported in part by a CNRS-ATP grant no. 2067, a NSERC grant no. 3667 and a FCAR grant no. 3578 .

\section{LITERATURE CITED}

Arao, T., Kawaguchi, A., Yamada, M. (1987). Positional distribution of fatty acids in lipids of the marine diatom Phaeodactylum tricornutum. Phytochemistry 26: 2573-2576

Badour, S. S., Gergis, M. S. (1965). Cell division and fat accumulation in Nitzschia sp. grown in continued illuminated mass culture. Arch. Microbiol 51. 94-102

Bligh, E. G., Dyer, W. J. (1959). A rapid method of total lipid extraction and purification. Can. J. Biochem. Physiol. 37. 911-917

Bloch, K., Constantopoulos, G., Kenyon, C., Nagai, J. (1967). Lipid metabolism of algae in the light and in the dark. In: Goodwin, T W. (ed.) Biochemistry of chloroplasts, Vol. 2. Academic Press, New York, p. 197-211

Brockman, U. H., Eberlein, K., Hentzschel, G., Schone, H. K., Siebers, D., Wandschneider, K., Weber, A. (1977). Parallel plastic tank experiments with cultures of marine diatoms. Helgoländer wiss. Meeresunters. 30: 201-216

Enright, C. T., Newkirk, G. F., Craigie, J. S., Castell, J. D. (1986). Growth of juvenile Ostrea edulis L. fed Chaetoceros gracilis of varied chemical composition. J. exp. mar. Biol. Ecol. 96: 15-26

Fogg, C. E. (1956). Photosynthesis and formulation of fats in a diatom. Ann. Bot. (N.S.) 20: 265-285

Goutz, M., Saliot, A. (1980). Relationship between dissolved and particulate fatty acids and hydrocarbons, chlorophyll $a$ and zooplankton biomass in Villefranche bay, Mediterranean sea. Mar. Chem. 8: 299-318

Grice, G. D., Reeve, M. R. (1982). Marine mesocosms. Biological and chemical research in experimental ecosystems. Springer-Verlag, New York

Harris, E., Riley, G. A. (1956). Oceanography of Long Island Sound. Chemical composition of the plankton. Bull. Bingham Oceanogr. Coll. 15: 315-325

Haug, A., Myklestad, S., Sakshaug, E. (1973). Studies on the phytoplankton ecology of the Trondheimfjord. I. The chemical composition of phytoplankton populations. J. exp. mar. Biol. Ecol. 11: 15-26

Holm-Hansen, O., Lorenzen, C. J., Holmes, R. W., Strickland, J. D. H. (1965). Fluorometric determination of chlorophyll. J. Cons perm. int. Explor. Mer 25: 115-128

Holz, G. G. (1981). Non-isoprenoid lipids and lipid metabolism of marine flagellates. In: Biochemistry and physiology of protozoa, Vol. 4. Academic Press, New York, p. 301-332

Jeffries, H. P. (1970). Seasonal composition of temperate plankton communities: fatty acids. Limnol. Oceanogr. 15: 419-426

Jeffries, H. P. (1972). Fatty acid ecology in a tidal marsh. Limnol Oceanogr 17: 433-440

Kattner, G., Gercken, G., Eberlein, K. (1983). Development of lipid during a spring bloom in the northern North sea. I. Particulate fatty acids. Mar. Chem. 14: 149-162

Lebart, L., Morineau, A., Tabard, N. (1977). Technique de la description statistique. Dunod Ed., Paris

Legendre, L., Legendre, P. (1984). Ecologie numérique. 2. La structure des données écologiques $2^{\mathrm{e}}$ edn. Masson, Paris

Lowry, O. H., Rosebrough, N. I., Farr, A. L., Randall, R. J. (1951). Protein measurement with Folin phenol reagent. J. Biol. Chem. 193: 265-275

Mayzaud, P., Ackman, R. G. (1976). Some empirical observations on the choice of carrier gas in the chromatographic analysis of fatty acid methyl-ester using well coated open tubular columns. Chromatographie 9: 321-324

Mayzaud, P., Chanut, J.-P., Ackman, R. G. (1989). Seasonal changes of the biochemical composition of marine particulate matter with special reference to fatty acid and sterols. Mar Ecol. Prog. Ser. 56: 189-204

Morris, R. J. (1984). Studies of a spring phytoplankton bloom in an enclosed experimental ecosystem. II. Changes in the component fatty acid and sterols. J. exp. mar. Biol. Ecol. 75: $59-70$

Morris, R. J., McCartney, M. J., Robinson, G. A. (1983). Studies of a spring phytoplankton bloom in an enclosed experimental ecosystem. I. Biochemical changes in relation to the nutrient chemistry of water. J. exp. mar. Biol. Ecol. 70: 249-262

Morris, R. J., McCartney, M. J., Joint, I. R., Robinson, G. A. (1985). Further studies of a spring phytoplankton bloom in an enclosed experimental ecosystem. J. exp. mar. Biol. Ecol. 86: 151-170

Morrison, W R., Smith, L. M. (1964). Preparation of fatty acid methylesters and dimethyl acetals from lipids with boron fluoride-methanol. J. Lipid Res. 5: 600-608

Mortensen, S. H., Borsheim, K. Y., Rainuzzo, J. R., Knutsen, G. (1988). Fatty acid and elemental composition of the marine diatom Chaetoceros gracilis Schütt. Effects of silicate deprivation, temperature and light intensity. J. exp. mar Biol. Ecol. 122: 173-185

Nichols, B. W. (1965). Light induced changes in the lipids of Chlorella vulgaris. Biochem. Biophys. Acta 106: 274-279

Nichols, B. W., Stubbs, J. M., James, A. T (1967). The lipid composition and ultrastructure of normal developing and degenerating chloroplasts. In: Goodwin, $T$ W (ed.) Biochemistry of chloroplasts, Vol. 2. Academic Press, New York, p. 677-690

Orcutt, D. M., Patterson, G. W. (1974). Effect of light intensity upon lipid composition of Nitzschia closterium. Lipids 9: $1000-1003$

Palmisano, A. C., Lizotte, M. P., Smith, G. A., Nichols, P. D., White, D. C., Sullivan, C. W (1988). Changes in photosynthetic carbon assimilation in Antarctic sea-ice diatoms during spring blooms: variation in synthesis of lipid classes. J. exp. mar. Biol. Ecol. 116: 1-13 
Parrish, C. C. (1987). Time series of particulate and dissolved lipid classes during spring phytoplankton blooms in Bedford Basin, a marine inlet. Mar. Ecol. Prog. Ser 35: $129-139$

Pöhl, P., Zurheide, F. (1979). Fatty acid and lipids of marine algae and the control of their biosynthesis by environmental factors. In: Hoppe, H. A., Levring, T., Yanaha, Y (eds.) Marine algae in pharmaceutical sciences. Walter de Gruyter, Berlin, p. 473-583

Redfield, A. C. (1934). On the proportions of organic derivatives in sea water and their relation to the composition of plankton. James Johnstone Memorial Volume (Liverpool), p. 176

Schultz, D. (1974). Source, formation and composition of suspended lipoidal material in Narragansett Bay, Rhode Island, Ph. D. thesis, Univ, of Rhode Island

This article was presented by Dr R. J. Conover, Dartmouth, N.S., Canada
Shifrin, W. S., Chisholm, S. W (1981). Phytoplankton lipids: interspecific differences and effects of nitrate, silicate and light-dark cycles. J. Phycol. 17: 374-384

Tréguer, P., Le Corre, P. (1974). Manuel d'analyses de sels nutritifs dans l'eau de mer. Utilisation de l'autoanalyseur II (2nd edn). Laboratoire d'océanographie chimique, Université de Bretagne Occidentale

Vorbeck, M. L., Marinetti, G. V (1965). Separation of glycosyl diglycerides from phosphatides using silicic acid column chromatography. J. Lipid Res. 6: 3-6

Wood, B. J. B. (1974). Fatty acid and saponifiable lipids. In: Stewart, D. W. (ed.) Algae physiology and biochemistry. Blackwell, Oxford, p. 236-265

Yentsch, C. S., Menzel, D. (1963). A method for the determination of phytoplankton chlorophyll and phaeophytin by fluorescence. Deep Sea Res. 10: 221-231

Manuscript first received: December 20, 1988 Revised version accepted: September 26, 1989 